\title{
kontakt.los!
}

\section{Bildung und Beratung für Familien während der Corona-Pandemie}

Doris Lüken-Klaßen

Regina Neumann

Susanne Elsas

\section{kontakt (II) los!}

Bildung und Beratung für Familien während der Corona-Pandemie. 
(c) 2020 Staatsinstitut für Familienforschung an der Universität Bamberg (ifb) 96045 Bamberg

Hausadresse: Heinrichsdamm 4, 96047 Bamberg

Leiterin: $\quad$ Prof. Dr. Henriette Engelhardt-Wölfler

Stellv. Leiter: Dipl.-Soz. Harald Rost

Tel.: $\quad$ (0951) 965250

Fax: $\quad$ (0951) $96525-29$

E-Mail: $\quad$ sekretariat@ifb.uni-bamberg.de

ISSN (Online): 2566-6061

DOI: $\quad 10.20378 /$ irb-48811

Jeder Nachdruck und jede Vervielfältigung - auch auszugsweise - bedürfen der ausdrücklichen Genehmigung des Staatsinstituts für Familienforschung an der Universität Bamberg. 


\section{Inhaltsverzeichnis}

Hintergrund: Die Corona-Pandemie belastet Familien - und beschränkt Unterstützungsmöglichkeiten............................................................................................5

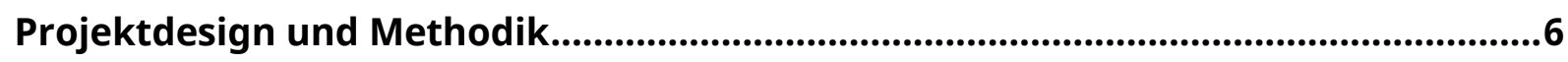

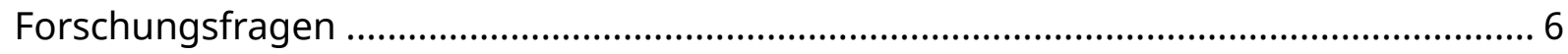

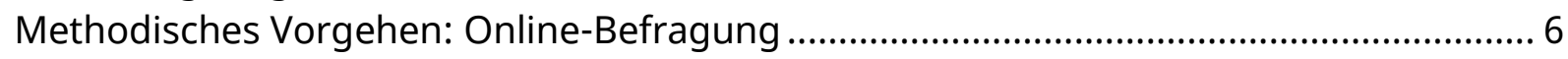

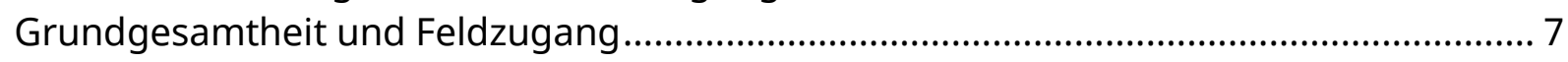

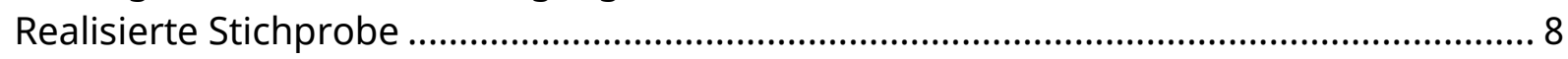

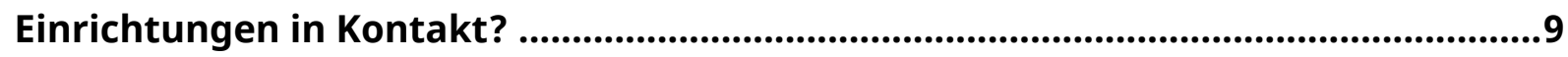

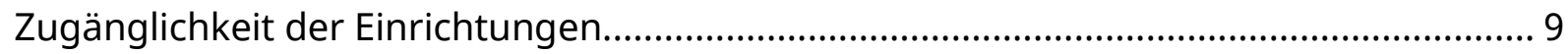

Zielgruppenerreichung und Gefährdungen ............................................................... 11

Veränderung finanzieller und personeller Ressourcen ..............................................15

Die Anliegen der Adressatinnen und Adressaten .......................................................21

Die Corona-Pandemie als Digitalisierungsmotor? ..................................................25

Digitale Formate: Während und vor der Pandemie .................................................... 25

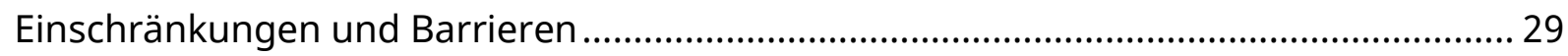

Good Practice: Erfahrungen während der Pandemie ..................................................... 32

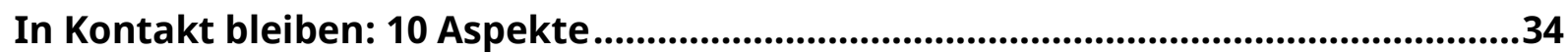

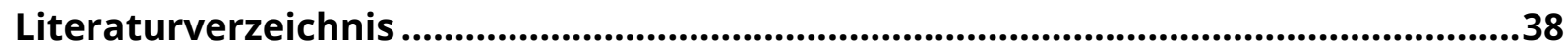




\section{Abbildungsverzeichnis}

Abbildung 1: Öffnung der Einrichtungen während der Pandemie im Mai 2020 .............. 10

Abbildung 2: Mittlere Anzahl der Eltern/Schwangeren pro Woche.................................. 11

Abbildung 3: Erreichung der Zielgruppe und Gefährdungspotenzial.............................. 13

Abbildung 4: Existenzbedrohung durch Einnahmeausfälle ............................................ 15

Abbildung 5: Existenzbedrohung der Familienbildungseinrichtungen ............................ 16

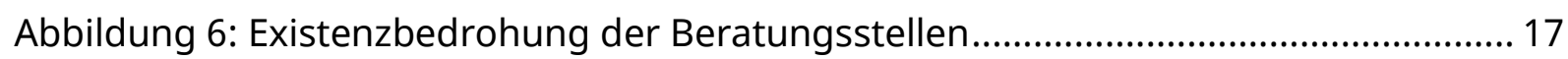

Abbildung 7: Kompensation der Einnahmeausfälle durch staatliche Hilfsprogramme.. 17

Abbildung 8: Kompensation der Einnahmeausfälle durch staatliche Hilfsprogramme.. 18

Abbildung 9: Personelle Ressourcen: Familienbildungseinrichtungen ........................... 19

Abbildung 10: Personelle Ressourcen: Beratungsstellen................................................. 19

Abbildung 11: Personelle Ressourcen: KoKis.................................................................... 20

Abbildung 12: Anliegen der Eltern/Schwangeren in Beratungsstellen............................ 21

Abbildung 13: Anliegen der Eltern/Schwangeren in KoKis ............................................. 22

Abbildung 14: Anliegen der Eltern/Schwangeren in Familienbildungseinrichtungen .... 23

Abbildung 15: Rangfolge der am häufigsten genannten Themen ................................... 24

Abbildung 16: Digitale Angebote: Telefonische Beratung/Hotline .................................. 25

Abbildung 17: Digitale Angebote: Beratung per E-Mail.................................................. 26

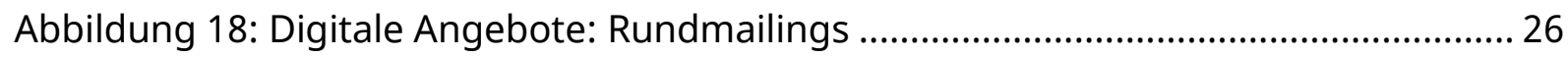

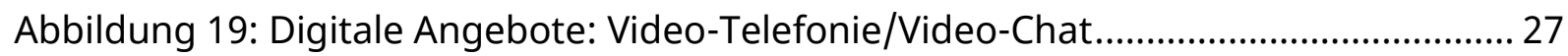

Abbildung 20: Digitale Angebote: Messenger-Broadcast-Liste/Gruppenchat................. 27

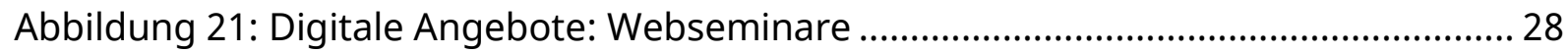

Abbildung 22: Digitale Angebote: Postings auf Facebook ................................................ 28

Abbildung 23: Als besonders bedauerlich bewertet wird die Absage von ..................... 30

Abbildung 24: Gründe für den Ausfall eines konkreten Angebotes................................... 30

Abbildung 25: Good Practices während der Corona-Pandemie ....................................... 32

Abbildung 26: Fortführung digitaler Good Practices auch nach der Corona-Pandemie. 33 


\section{Hintergrund: Die Corona-Pandemie belastet Familien - und beschränkt Unterstützungsmöglichkeiten}

Die Maßnahmen zur Eindämmung der Corona-Pandemie stellen Familien vor besondere Herausforderungen. Durch die Schließung von Betreuungs- und Bildungseinrichtungen sowie weitreichende Kontakt- und Ausgangsbeschränkungen hat der Familienalltag seit März 2020 eine neue Realität bekommen. Dieser musste in fast allen Familien tiefgreifend umgestaltet und reorganisiert werden. Solche Brüche mit dem gewohnten Alltag können positive Veränderungen hervorbringen. Wenn Mitglieder eines Familienhaushaltes etwa deutlich mehr Zeit miteinander verbringen (müssen), eröffnet dies neue Möglichkeiten für ein familiales Miteinander. Der neue Familienalltag geht aber auch mit zusätzlichen Anforderungen einher: Vereinbarkeit heißt nun für die meisten Eltern, Kinderbetreuung, Home-Schooling und die eigene Berufstätigkeit parallel managen zu müssen. Das findet nicht nur unter verschiedenen, branchen- und jobspezifischen Rahmenbedingungen statt, sondern stellt Mütter und Väter auch vor neue pädagogisch-erzieherische Herausforderungen. Bei vielen Familien kommen Sorgen um die eigene Gesundheit und die von Familienmitgliedern oder finanzielle Belastungen und Existenzängste hinzu. Derartige Belastungen können zu einem Anstieg von Partnerschafts- und Familienkonflikten und innerfamilialer Gewalt führen. Alle diese Aspekte verändern die Unterstützungs- und Orientierungsbedarfe von Eltern und Kindern. ${ }^{1}$

Eine große Rolle spielen hier die Bildungs-, Beratungs- und Vernetzungsangebote im Rahmen der Kinder- und Jugendhilfe: die Einrichtungen der Eltern- und Familienbildung (nachfolgend kurz: Familienbildung), der Frühen Hilfen sowie Beratungsstellen. Diese unterstützen Mütter, Väter und andere Erziehende dabei, ihre Erziehungsaufgaben gut wahrnehmen und ihren Familienalltag meistern zu können, und sie unterstützen Schwangere und Eltern bei der Bewältigung individueller und familienbezogener Probleme, der Lösung von Erziehungsfragen sowie bei Trennung und Scheidung. Zudem zeigen sie Wege auf, wie Konfliktsituationen in der Familie gewaltfrei gelöst werden können.

All diese Angebote finden meist im persönlichen Kontakt und häufig im Gruppensetting statt und mussten zwecks Eindämmung der Pandemie reduziert oder umstrukturiert werden; manche Einrichtungen mussten gar komplett schließen. Dadurch fallen Einnahmequellen weg, was bis zur Zahlungsunfähigkeit führen kann. Zudem ist das Personal teilweise selbst von Vereinbarkeitsproblemen durch Kita- und Schulschließungen betroffen. Die Fachkräfte stehen bei der Anpassung an veränderte Rahmenbedingungen somit vor großen Herausforderungen.

Es ist also eine Situation entstanden, die auf der einen Seite durch einen veränderten, teils dringlicheren Bedarf der Familien, auf der anderen Seite durch ein eingeschränktes Angebot und größere Zugangshürden zur (Konflikt-)Beratung gekennzeichnet ist. Mithin ist zu erwarten, dass angemessene Unterstützung nicht in ausreichendem Umfang angeboten werden kann.

1 Andresen et al. (2020); Bünning et al. (2020); Deutscher Ethikrat (2020); Erziehungshilfefachverbände (2020); Fatke et al. (2020); Mairhofer et al. (2020); StMAS (2020); Steinert/Ebert (2020). 


\section{Projektdesign und Methodik}

Ausgehend von der Frage, welche Folgen die Corona-Pandemie für das Familienleben, für die Eltern- und Familienbildung sowie für die Beratungslandschaft hat, initiierte das Staatsinstitut für Familienforschung an der Universität Bamberg (ifb) mit Unterstützung durch das Bayerische Staatsministerium für Familie, Arbeit und Soziales (StMAS) im Frühjahr 2020 das empirische Forschungsprojekt "kontakt.los! Bildung und Beratung für Familien während der Corona-Pandemie".

\section{Forschungsfragen}

Im Fokus der Untersuchung steht die Frage, wie sich die Maßnahmen zur Eindämmung von Covid-19 auf Einrichtungen der Familienbildung und Beratung auswirken und wie sich die Nachfrage und Bedarfe nach deren Angeboten verändern. Unser Hauptaugenmerk gilt Müttern, Vätern und anderen Erziehungsberechtigten; im Blick haben wir zudem Angebote für Schwangere und werdende Väter, für andere Familienmitglieder (z. B. Großeltern) sowie für junge Menschen allgemein. Unsere Forschungsfragen lauten:

- Inwieweit haben sich das Angebot an Familienbildung und das Angebot an Beratungsleistungen für Familien durch die Maßnahmen zur Eindämmung der CoronaPandemie verändert?

- Wie verändert sich der Handlungsspielraum der Einrichtungen, insbesondere die finanzielle und personelle Situation?

- Welche Formate und Themen fallen während der Corona-Pandemie weg?

- Welche neuen, digitalen Formate konnten während der Corona-Pandemie kreiert und umgesetzt werden?

- Können die neuen Angebote die wegfallenden Angebote kompensieren?

- Welche innovativen Angebote haben sich in der Krisenzeit bereits bewährt und sollten beibehalten oder weiter ausgebaut werden?

- Inwieweit ändern sich im Zuge der Corona-Pandemie die Bedarfe von Familien und ihre Nachfrage nach unterstützenden Angeboten?

- Wie gestalten und verändern sich Kontakthäufigkeit, -art und -inhalt sowie Merkmale der Familien während der Corona-Pandemie im Vergleich zu der Zeit vor der Corona-Pandemie?

- Welche Bedarfe bleiben wegen weggefallener Angebotsformate und veränderter Rahmenbedingungen ungedeckt?

- Welche Bedarfe können mit den neuen Formaten besser oder überhaupt erst gedeckt werden?

\section{Methodisches Vorgehen: Online-Befragung}

Zur Beantwortung obenstehender Fragen entwickelte das ifb eine quantitative OnlineBefragung von Einrichtungen der Familienbildung und Beratung aus ganz Bayern. ${ }^{2}$ Die

2 Unser Dank gilt Jan-Hendrik Kötting für die Programmierung der Online-Erhebung und seine Unterstützung bei der Datenaufbereitung. 
Befragung, programmiert mit der Umfrage-Software Unipark von Questback, beinhaltete Fragenkomplexe zu

- allgemeinen Merkmalen der Einrichtung,

- Präsenzangeboten und digitalen Angeboten vor und während der Pandemie,

- die Anliegen der Eltern und Schwangeren vor und während der Pandemie,

- Good Practices sowie Einschränkungen und Barrieren bei der Umsetzung innovativer Projekte und

- Veränderungen finanzieller und personeller Ressourcen.

Im Frühjahr 2020 war das gesellschaftliche Leben aufgrund der laufenden Anpassungen der Corona-Beschränkungen an das Infektionsgeschehen von großer Dynamik geprägt; auf den im März verhängten Lockdown folgten ab Ende April schrittweise Lockerungen. Damit die Antworten vergleichbar sind, sollten die Fachkräfte bei Fragen, die sich auf die Situation während der Pandemie beziehen, jeweils den Monat Mai 2020 fokussieren.

Die Fragenkomplexe basieren größtenteils auf Instrumenten, die sich bei früheren Befragungen des ifb etabliert haben; andere wurden für diese Befragung entwickelt. Die Antwortkategorien waren in der Regel geschlossen, doch es gab auch mehrere offene Antwortmöglichkeiten. Die Befragung war für eine Dauer von rund einer halben Stunde konzipiert, was auch der mittleren Bearbeitungszeit entsprach. ${ }^{3}$

\section{Grundgesamtheit und Feldzugang}

Es wurde eine Vollerhebung aller Beratungsstellen, Einrichtungen der Familienbildung sowie der Frühen Hilfen durchgeführt, die als anerkannte öffentliche und freie Träger der Kinder- und Jugendhilfe sowie der Erwachsenenbildung in Bayern tätig sind:

- Beratungsstellen:

- Beratungsstellen für Schwangerschaftsfragen

- Erziehungsberatungsstellen

- Ehe-, Partnerschafts-, Familien- und/oder Lebensberatungsstellen

(nachfolgend kurz: Ehe- und Familienberatungsstellen)

- Einrichtungen der Familienbildung:

- Familienbildungsstätten

- Mütter- und/oder Familienzentren

- Familienstützpunkte

- Frühe Hilfen: Koordinierende Kinderschutzstellen „KoKi - Netzwerk frühe Kindheit” (nachfolgend kurz: KoKis) ${ }^{4}$

Die meisten Einrichtungen lassen sich einem dieser sieben Einrichtungstypen zuordnen. Einige Einrichtungen vereinigen auch mehrere Einrichtungstypen unter einem Dach, beispielsweise eine Beratungsstelle, die sowohl Ehe- und Familienberatungsstelle als auch

3 Ermittelt wurde die mittlere Bearbeitungszeit derjenigen, die den Fragebogen ohne Unterbrechung beendet haben (Median: 29 Minuten, arithmetisches Mittel: 34 Minuten).

4 Die Koordinierenden Kinderschutzstellen (KoKi) bieten (werdenden) Eltern mit Kindern im Alter von 0 bis 3 Jahren in belastenden Lebenslagen und Überforderungssituationen kostenfreie und anonyme Beratung und Unterstützung an; sie sind beim Jugendamt angesiedelt. 
Beratungsstelle für Schwangerschaftsfragen ist, oder eine Familienbildungsstätte, die gleichzeitig ein Familienstützpunkt ist. Diese Einrichtungen wurden für die kontakt.los!Befragung nicht als zwei verschiedene, sondern als eine Einheit gezählt und angeschrieben. Insgesamt lagen 719 gültige E-Mail-Adressen von Einrichtungen aus ganz Bayern vor.

Anfang Juni 2020 haben diese Institutionen vom ifb Informationen über das Projekt und einen personalisierten Einladungslink zur Online-Befragung per E-Mail erhalten. Der Befragungszeitraum erstreckte sich über vier Wochen bis Anfang Juli 2020. Diejenigen, welche die Befragung nicht abgeschlossen hatten, erhielten zwischenzeitlich per E-Mail ein Erinnerungsschreiben. Auch die Dachverbände ${ }^{5}$ wurden über das Projekt informiert; mehrere dieser warben bei ihren Mitgliedern um die Teilnahme an der Befragung.

\section{Realisierte Stichprobe}

Die Grundgesamtheit lag bei N = 719. Von diesen haben sich 402 Einrichtungen, also über die Hälfte, an der Befragung beteiligt; die Ausschöpfungsquote liegt bei $56 \%$.

Sowohl die Anzahl als auch die Teilnahmequote unterscheiden sich nach Einrichtungstyp. Von allen liegen jedoch ausreichend hohe Fallzahlen vor, sodass Vergleiche zwischen den Einrichtungstypen möglich sind. Das Gleiche gilt für die Trägerschaft: Beteiligt haben sich sowohl Einrichtungen in freier als auch solche in öffentlicher Trägerschaft (218 bzw. 182), sowohl große als auch kleine Einrichtungen. Auch hinsichtlich politisch-geographischer Aspekte ist der Rücklauf ausgewogen: Aus ganz Bayern - aus allen sieben Regierungsbezirken - haben sich Einrichtungen an der Befragung beteiligt, die sich sowohl in kreisfreien Städten als auch in Landkreisen verorten.

Dank der breiten Beteiligung kann somit ein umfassendes Bild über die Situation der Einrichtungen gezeichnet werden. Wie bei allen Datenerhebungen mit freiwilliger Teilnahme muss bei der Interpretation der Daten jedoch eine potenzielle Verzerrung der Ergebnisse aufgrund systematisch unterschiedlicher Teilnahmebereitschaft berücksichtigt werden: Einige Einrichtungen waren zum Befragungszeitraum komplett geschlossen; manche standen vor gravierenden finanziellen Problemen und/oder befanden sich in einer sehr angespannten Personal- und Arbeitssituation. Es ist möglich, dass diejenigen, die vor besonders großen Herausforderungen standen, überproportional häufig an der Befragung teilnahmen, um auf ihre problematische Situation aufmerksam zu machen. Vorstellbar ist aber auch, dass sich diese Einrichtungen besonders selten an der Befragung beteiligten, weil die Prioritäten anders gesetzt werden mussten. Zudem erscheint plausibel, dass sich diejenigen Einrichtungen, die es während der Pandemie besonders gut geschafft haben, Kontakt zu Familien zu halten, überproportional häufig an der Befragung beteiligt haben, um ihre Erfolge teilen und weitergeben zu können.

Landesarbeitsgemeinschaft (LAG) der Bayerischen Familienbildungsstätten, Landesverband der Mütterund Familienzentren, Landesarbeitsgemeinschaft und Fachverband für Erziehungs-, Jugend- und Familienberatung in Bayern (LAG Erziehungsberatung), Landesarbeitskreis für Ehe-, Partnerschafts-, Familienund Lebensberatung (LAK-EPFLB). 


\section{Einrichtungen in Kontakt?}

Professionelle Bildungs-, Beratungs- und Vernetzungsangebote im Rahmen der Kinderund Jugendhilfe sollen Orientierungsbedarfe von Kindern und Jugendlichen, von Schwangeren und Müttern, Vätern sowie anderen Erziehenden decken, Familien dabei unterstützen, dass Kinder gesund aufwachsen können, dass der Familienalltag gut und gewaltfrei gelingt sowie Krisen und schwierige Lebenslagen bewältigt werden können.

Im Rahmen ihres Aufgabenspektrums haben die Einrichtungen der Familienbildung und Beratung den gesellschaftlichen Auftrag, für alle Familien da zu sein. Daher sind der persönliche, direkte Kontakt, gute Erreichbarkeit und niedrigschwellige Zugangsmöglichkeiten wichtige Prinzipien ihrer Arbeit.

Ausgelöst durch die Maßnahmen zur Eindämmung der Corona-Pandemie sind die Einrichtungen jedoch nicht auf dieselbe Weise zugänglich wie zuvor: Da die Angebote von Familienbildungseinrichtungen, Frühen Hilfen und Beratungsstellen vor allem im persönlichen Kontakt - und häufig im Gruppen-Setting - stattfinden, mussten diese ihre Angebote reduzieren oder umstrukturieren; manche waren von vollständigen Schließungen betroffen.

\section{Zugänglichkeit der Einrichtungen}

Der Zugang zu den Einrichtungen war zu Beginn der Corona-Pandemie stark eingeschränkt, und zwar sowohl während des Lockdowns im März und April als auch darüber hinaus: Im Mai 2020 traten zwar schrittweise Lockerungen in Kraft, weil aber Kontaktbeschränkungen, Abstandsregelungen und Hygieneauflagen anhielten bzw. neu eingeführt wurden, blieb die physische Zugänglichkeit eingeschränkt.

Bei einem Drittel (33 \%) der Einrichtungen blieben die Türen auch im Mai 2020 für Familien komplett verschlossen; knapp zwei Drittel (66 \%) der befragten Einrichtungen konnten nur mit Einschränkungen betreten werden. Rund $1 \%$ der Fachkräfte gab an, dass Familien uneingeschränkt in die Einrichtung kommen konnten.

Die Zugänglichkeit unterscheidet sich je nach Einrichtungstyp: Die Beratungsstellen also Ehe- und Familienberatungsstellen, Erziehungsberatungsstellen und Beratungsstellen für Schwangerschaftsfragen - waren im Mai 2020 zu rund einem Fünftel (18\%) für Schwangere und Eltern nicht zugänglich. KoKis waren zu einem Viertel (26\%) nicht zugänglich. Im Bereich der präventiven Familienbildung - also bei Familienbildungsstätten, Mütter- und Familienzentren und Familienstützpunkten - ist dieser Anteil deutlich höher: Mehr als die Hälfte (56 \%) der Familienbildungseinrichtungen gab an, dass Familien im Mai 2020 nicht in ihre Einrichtungen kommen konnten (vgl. Abbildung 1). ${ }^{6}$

6 Die Unterschiede zwischen den Einrichtungen desselben Typs können bedingt sein durch lokal unterschiedliche Praxis bezüglich Sprechzeiten, durch die unterschiedliche personelle, finanzielle und räumliche Ausstattung, durch lokal verschiedenes Pandemie-Geschehen sowie verschiedene politische Entscheidungen vor Ort. 
Abbildung 1: Öffnung der Einrichtungen während der Pandemie im Mai 2020

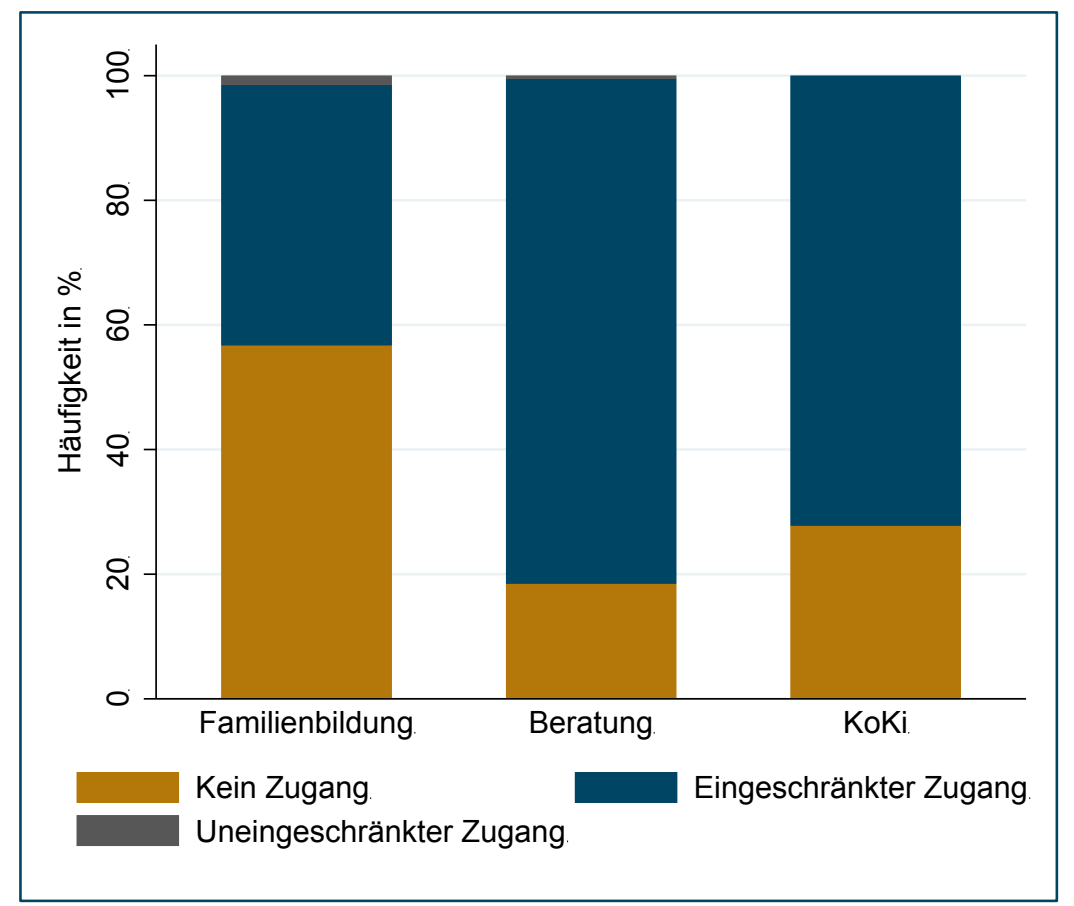

Quelle: ifb-Befragung „kontakt.los! 2020” ( $=$ 402; keine Angabe = 15; "andere“ Einrichtungen = 4)

Eine unterschiedliche Teilnahmebereitschaft bei Umfragen kann Ergebnisse verzerren (siehe Kapitel: Realisierte Stichprobe). Diese Verzerrung ist dann systematisch und für die Interpretation der Ergebnisse relevant, wenn die Teilnahmebereitschaft davon abhängt, zu welchem Thema die Befragung durchgeführt wird. So ist zu erwarten, dass die Bereitschaft, an einer Befragung teilzunehmen, welche das Kontakthalten sowie digitale Angebote während der Corona-Pandemie fokussiert, bei eben jenen Einrichtungen gering ist, denen es nicht gelungen ist, mit den Familien in Kontakt zu bleiben. Für die hier vorgestellten Zahlen bedeutet dies: Es muss davon ausgegangen werden, dass der Anteil der Einrichtungen, die für Familien nicht zugänglich waren, in der Gesamtheit der Einrichtungen höher war als das hier genannte Drittel.

Ein positives Ergebnis ist, dass von den 124 befragten Einrichtungen, die für Eltern nicht zugänglich waren, nahezu alle (96\%) trotzdem Angebote bereitstellen konnten. Insgesamt waren somit 99 \% der Einrichtungen, die sich an der Befragung beteiligt haben, im Monat Mai 2020 in Kontakt mit Familien - und somit nicht "kontaktlos".

Die Anzahl der Kontakte hat im Zuge der Pandemie gleichwohl massiv abgenommen. In den offenen Angaben merkten zahlreiche Fachkräfte an, dass es aufgrund der veränderten Angebote und der erschwerten Zugänglichkeit für Familien insgesamt schwieriger geworden ist, die Einrichtungen aufzusuchen. Und so lag die Anzahl der Eltern und Schwangeren, die pro Woche die Beratungs- und Familienbildungsangebote der Einrichtungen nutzten, im Mai 2020 bei allen Einrichtungstypen deutlich unter dem sonst üblichen Wert. Im Durchschnitt ging die Anzahl um mehr als die Hälfte zurück, sodass durchschnittlich nicht einmal halb so viele Eltern und Schwangere erreicht wurden wie im Jahr 2019, also vor der Pandemie. 
Das Ausmaß des Rückgangs variierte nach Einrichtungstyp; besonders gravierend fiel er bei den Familienbildungseinrichtungen und KoKis aus: Bei Einrichtungen der Familienbildung reduzierte sich die mittlere Anzahl an Eltern und Schwangeren, die pro Woche die Einrichtungen aufsuchen, im Zuge der Pandemie auf ein Fünftel; bei den KoKis reduzierte sich die mittlere Anzahl auf ein Drittel (vgl. Abbildung 2). ${ }^{7}$

Abbildung 2: Mittlere Anzahl der Eltern/Schwangeren pro Woche, vor und während der Pandemie

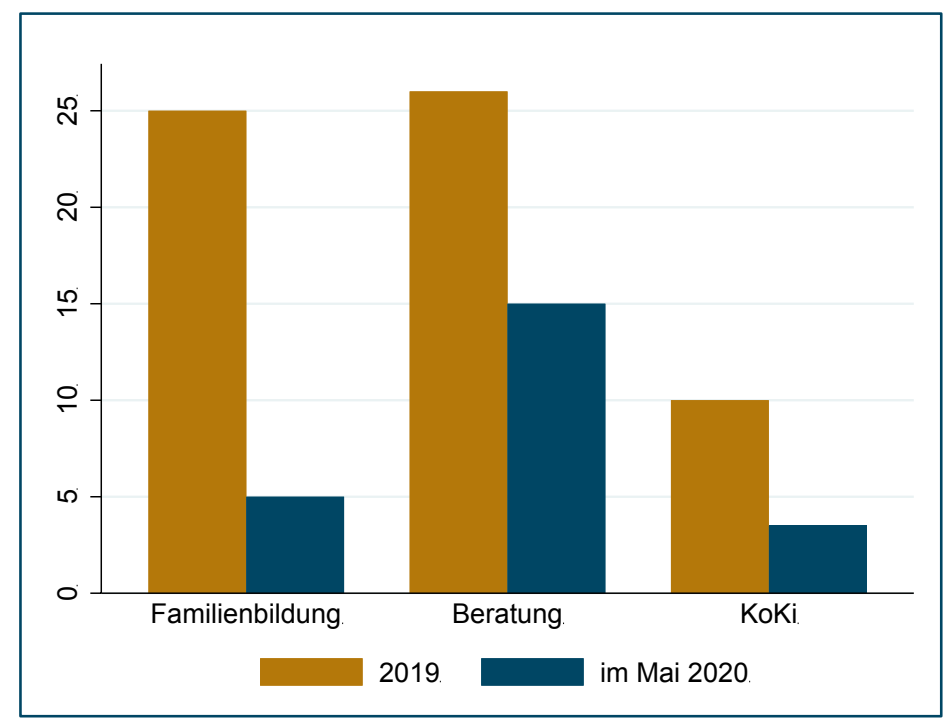

Quelle: ifb-Befragung „kontakt.los! 2020” ( $=$ 402; keine Angabe: "2019“= 83; "Im Mai 2020“= 84) Anmerkung: Verwendetes Maß für die "mittlere Anzahl”: Median

Bei den Beratungsstellen fällt der Rückgang etwas moderater aus, aber auch bei diesen halbierte sich die mittlere Anzahl der Ratsuchenden nahezu (vgl. Abbildung 2). Schwangerschaftsberatungsstellen und Erziehungsberatungsstellen verzeichnen dabei einen stärkeren Rückgang als Ehe- und Familienberatungsstellen.

\section{Zielgruppenerreichung und Gefährdungen}

Die Zielgruppen der Familienbildung und Beratung werden aufgrund der Pandemie derzeit nicht so erreicht wie üblich. Dies liegt nicht nur an der eingeschränkten Zugänglichkeit der Einrichtungen. Die Ursachen für den geringeren Kontaktumfang sind auch bei den Familien und ihren aktuellen Lebensumständen zu finden. So holen sich

7 Die meisten Einrichtungen sind eher klein. Diesen stehen einige wenige sehr große Einrichtungen gegenüber, die meist in Großstädten liegen, deutlich mehr Angebote durchführen und somit eine höhere Anzahl an Menschen erreichen. Hier wurde nun nicht das jeweilige arithmetische Mittel („Durchschnittswert") dargestellt, sondern der Median, der gegenüber Extremfällen robuster ist und Veränderungen somit deutlicher aufzeigen kann. Zu verstehen sind die Zahlen wie folgt: Vor der Pandemie wurden die Angebote der Familienbildungseinrichtungen pro Woche im Mittel von 25 Teilnehmenden genutzt - das heißt, die Hälfte der Familienbildungseinrichtungen hatte wöchentlich 25 Teilnehmende oder weniger, die andere Hälfte hatte (oft deutlich) mehr als 25. Während der Pandemie sank dieser Mittelwert (Median) auf 5. Zu beachten ist, dass das jeweilige arithmetische Mittel (die „durchschnittlichen“ Teilnahmezahlen) deutlich höher ausfällt als der hier dargestellte Median. 
coronabedingt weniger Eltern und Schwangere professionelle Hilfe, so die Einschätzung von mehr als die Hälfte (54 \%) der Fachkräfte (vgl. Abbildung 3).

Im Fragebogen gab es die Möglichkeit, zusätzliche Aspekte zu nennen, die sich aufgrund der Corona-Pandemie verändert haben. Zahlreiche Fachkräfte $(n=70)$ nutzten dieses Kommentarfeld. Eine Fachkraft notierte: „Im vergangenen Katastrophen-Zeitraum war es sehr, sehr ruhig (wenig Anfragen). Sehr verwunderlich.“ 26 Personen betonten, dass die Zugänge derzeit schwieriger seien. So wurde berichtet, dass vor allem der Anteil an Menschen, die das erste Mal die Einrichtung aufsuchen, bemerkenswert gering sei. Kannten Familien die Einrichtung bereits, sei die Kontaktpflege eher zu bewerkstelligen als der Aufbau von Kontakten mit neuen Familien: „Bestehende Kontakte ließen sich gut halten, neue Kontakte eher problematisch." Dies gelte auch für frischgebackene Eltern: „Aufgrund von Corona erreichen wir alle unsere Adressatinnen sehr schlecht, gerade auch der Zugang für neue Eltern mit kleinen Babys, die uns noch nicht von vor Corona kannten, ist schwierig."

Die Hemmschwellen scheinen also höher zu sein, und einige Eltern kommen, wie eine Fachkraft feststellte, "häufig erst, wenn sich die Situation schon sehr zugespitzt hat", und die "Familien, die kommen, sind psychisch hoch belastet!"Wie mehrere Fachkräfte übereinstimmend berichteten, haben diejenigen, die den Weg in die Einrichtungen suchen - auch diejenigen, die vorher bereits zur Klientel gehörten -, mehr Beratungsbedarf als zuvor. 18 Fachkräfte merkten an, dass sich die Problemlagen aufgrund der Corona-Pandemie verschärfen und beispielsweise finanzielle Probleme, Ängste, Sorgen und Depressionen zunehmen. Es gebe schlicht Redebedarf (,viel Zeit nimmt der Teil in Anspruch, in dem Eltern beschreiben, wie sich durch Corona ihr Familienleben verändert hat"), und dies liege daran, dass die Inhalte „extrem individuell" seien. Mehrere Fachkräfte merkten an: „Mehrfachbelastungen sind fast immer Thema. " Die Problemlagen seien komplexer und coronabedingt „potenzieren sich bestehende Probleme und mangelnde personale, soziale und materielle Ressourcen". 53 \% der Befragten stimmten der Aussage zu, dass Eltern coronabedingt häufiger mit der Erziehung überfordert scheinen (vgl. Abbildung 3).

Vor diesem Hintergrund ist im Blick zu behalten, dass fast jede vierte Fachkraft (23\%) der Aussage zustimmte, dass die Einrichtung den Bedarfen der Familien aufgrund der aktuellen Umstände nicht mehr gerecht werden kann (vgl. Abbildung 3). Sorge bereitet mehreren Fachkräften zudem, dass einige Konflikte unter der Oberfläche schwelen und erst allmählich aufbrechen. Zum Beispiel sei bei getrenntlebenden Eltern häufiger das "Umgangsrecht schwierig" und "Krisen [treten] erst jetzt nach akuter Corona-Zeit" zu Tage. Eine andere Fachkraft schrieb, die „Probleme [sind] immer noch verdeckt, sie kommen (noch) nicht ans Licht. V. a. Mittelschicht-Familien holen sich keine Hilfe, sie haben den Anspruch, alles selber zu schaffen."

Die Zugänglichkeit scheint allerdings insbesondere für diejenigen Gruppen erschwert, welche die Unterstützung besonders nötig hätten: „Aufgrund der Corona-Pandemie erreichen wir Eltern und Schwangere in belasteten Lebenslagen schlechter", so die Einschätzung von mehr als zwei Dritteln (69 \%) der befragten Fachkräfte (vgl. Abbildung 3). Durch die Pandemie sei die "Klientel stark verunsichert - handlungsgebremst". In offenen Antworten öfter erwähnt wurden speziell die Alleinerziehenden, die vom Lockdown sowie von (zeitweisen) Schließungen von Bildungs- und Betreuungseinrichtungen besonders getroffen waren bzw. sind. Des Weiteren wurde auf bildungsinstitutionenferne Familien 
hingewiesen: "Man erreicht eine bestimmte Zielgruppe an Eltern (höhere Bildungsabschlüsse), bildungsfernere Familien sind untergetaucht und auch nicht über digitale Wege zu erreichen."

\section{Abbildung 3: Erreichung der Zielgruppe und Gefährdungspotenzial}

\section{Aufgrund der Corona-Pandemie}

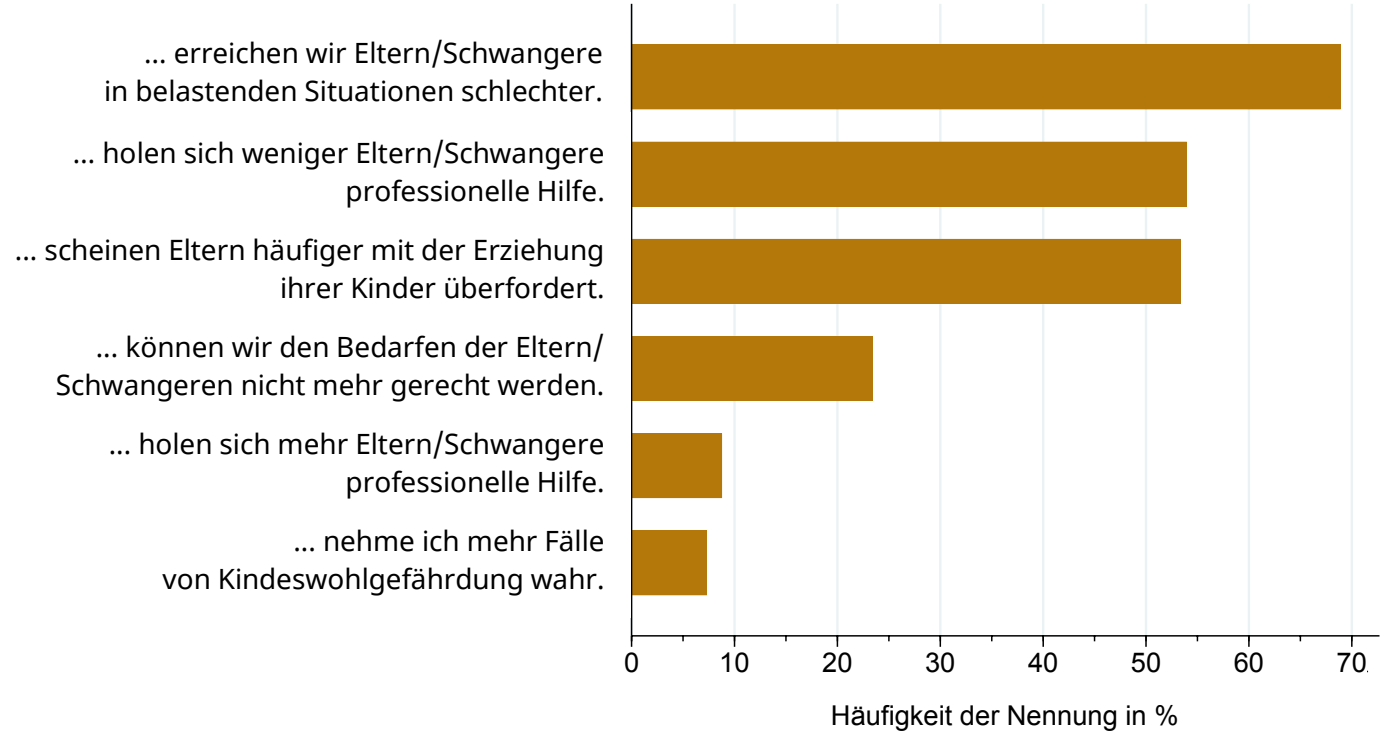

Quelle: ifb-Befragung "kontakt.los! 2020" ( $n=402$; keine Angabe = 61);

Anmerkung: Zustimmungswerte in \%, Mehrfachnennungen möglich

Auch Familien mit Migrationshintergrund stellen derzeit einen durchschnittlich kleineren Anteil der Teilnehmerstruktur bzw. der Klientel dar als üblicherweise: Machten diese 2019 im Mittel einen Anteil von 35 \% aus, so waren es im Mai 2020 nur noch 26 \%. Als Erklärung gab eine Fachkraft an, diese Familien "möchten den persönlichen Kontakt; telefonieren oder Online-Beratung ist für sie schwieriger". Insbesondere ist der Kontakt zu Familien mit Fluchtgeschichte erschwert: „Aufgrund von Corona erreichen wir Eltern/Schwangere ohne deutsche Sprachkenntnisse, z. B. Asylsuchende, schlechter." Bei ihnen kommt zu bereits genannten Problemen hinzu, dass aufsuchende Angebote wegfallen, weil der Zugang zu Unterkünften nicht möglich ist: „Die GUs [Gemeinschaftsunterkünfte] und Ankerzentren [Ankunfts-, Entscheidungs- und Rückführungseinrichtungen] sind sehr abgeschottet, dort kam fast jede Hilfe zum Stillstand, die Beschulung der Kinder ist eine Katastrophe."

Zur Kindeswohlgefährdung werden unterschiedliche Erfahrungen gemacht: Nach Einschätzung einiger Fachkräfte nimmt diese zu: 25 Befragte (7\%) bestätigen, dass sie coronabedingt mehr Fälle von Kindeswohlgefährdungen wahrnehmen (vgl. Abbildung 3). Hier lassen sich deutliche Unterschiede nach dem Einrichtungstyp ausmachen: Von den Fachkräften der Familienbildung stimmten 6 \% zu, von den KoKi-Fachkräften $10 \%$. Und während nur $2 \%$ der Schwangerschaftsberatungen mehr Fälle von Kindeswohlgefährdungen aufgrund der Corona-Pandemie wahrnahmen, traf dies bei $6 \%$ der Ehe- und Familienberatungsstellen und $18 \%$ der Erziehungsberatungsstellen zu.

Dass im Durchschnitt 7 \% der Befragten mehr Kindeswohlgefährdungen wahrnehmen, bedeutet im Umkehrschluss auch, dass das Gros der Fachkräfte keine Zunahme der 
Kindeswohlgefährdung beobachtet. So beruhigend dies anmuten mag, kann dieser Erfahrungswert daran liegen, dass Fälle von Kindeswohlgefährdungen im Referenzzeitraum nicht sichtbar werden (konnten) - wie mehrfach von Fachkräften thematisiert wird: "Eltern (...) sind abgetaucht" und "gefährdete Kinder sind von [der] Bildfläche verschwunden. Die Familien auch. "Durch die Schließung und den eingeschränkten Betrieb von Bildungsund Betreuungseinrichtungen sind die sonst wirksamen Präventions- und Meldeketten bei Verdacht auf Kindeswohlgefährdung unterbrochen. Eine Fachkraft alarmiert, aufgrund der Corona-Pandemie gibt es „in beunruhigendem Maß keine externen Anfragen von Kindertageseinrichtungen zu 8a-Beratung ${ }^{8}$ (iseF), d. h. die Kinder sind weniger im Blick".

8 Gemeint ist der § 8a SGB VIII: Schutzauftrag bei Kindeswohlgefährdung, nach welchem bei einem Verdacht auf Kindeswohlgefährdung eine Fachkraft mit spezieller Zusatzausbildung - eine insoweit erfahrene Fachkraft (iseF) - hinzugezogen wird, um das Gefährdungsrisiko einzuschätzen. 


\section{Veränderung finanzieller und personeller Ressourcen}

Die Maßnahmen zur Bekämpfung der Corona-Pandemie wirken sich auf die Angebote und den Betrieb der Einrichtungen aus. Mit dem Wegbleiben der Teilnehmenden sind auch Kurs- und Veranstaltungsgebühren weggefallen; ferner mussten Feste und Aktionen abgesagt werden, sodass auch auf diesem Wege keine Einnahmen generiert werden konnten. Auch die zukünftige finanzielle Förderung schien für einige nicht gesichert. Da es sich zudem um gemeinnützige Einrichtungen handelt, besitzen sie in der Regel keine nennenswerten Rücklagen, mit denen diese Einbußen abgefedert werden können.

Daher wurde um eine Einschätzung ihrer Existenzbedrohung aufgrund wegfallender Einnahmen gebeten. Wie existenzbedrohend der Wegfall erwarteter Einnahmen für die Einrichtung war, konnte auf einer 11-er Skala zwischen „überhaupt nicht existenzbedrohend" (1) und „voll und ganz existenzbedrohend" (11) eingeschätzt werden. Weil nicht alle Einrichtungen Einkommenseinbußen zu verzeichnen hatten, gab es darüber hinaus die Möglichkeit, „trifft nicht zu“ zu wählen.

Die Koordinierenden Kinderschutzstellen haben diese Frage nicht erhalten, weil sie in der öffentlichen Kinder- und Jugendhilfe direkt beim örtlichen Jugendamt installiert sind und aufgrund ihrer Finanzierungsstruktur keine existenzbedrohenden Einnahmeverluste erlitten haben können. Von denjenigen Einrichtungen, die diese Frage erhalten haben, wählten $14 \%$ die Möglichkeit „trifft nicht zu“. Zusammengenommen kann somit festgehalten werden, dass rund $28 \%$ der Einrichtungen keine Einnahmeausfälle zu verzeichnen hatten. Rund ein Drittel der Befragten hat diese freiwillige Frage nicht beantwortet, $37 \%$ der Einrichtungen gaben eine Einschätzung ihrer Existenzbedrohung ab.

Abbildung 4: Existenzbedrohung durch Einnahmeausfälle

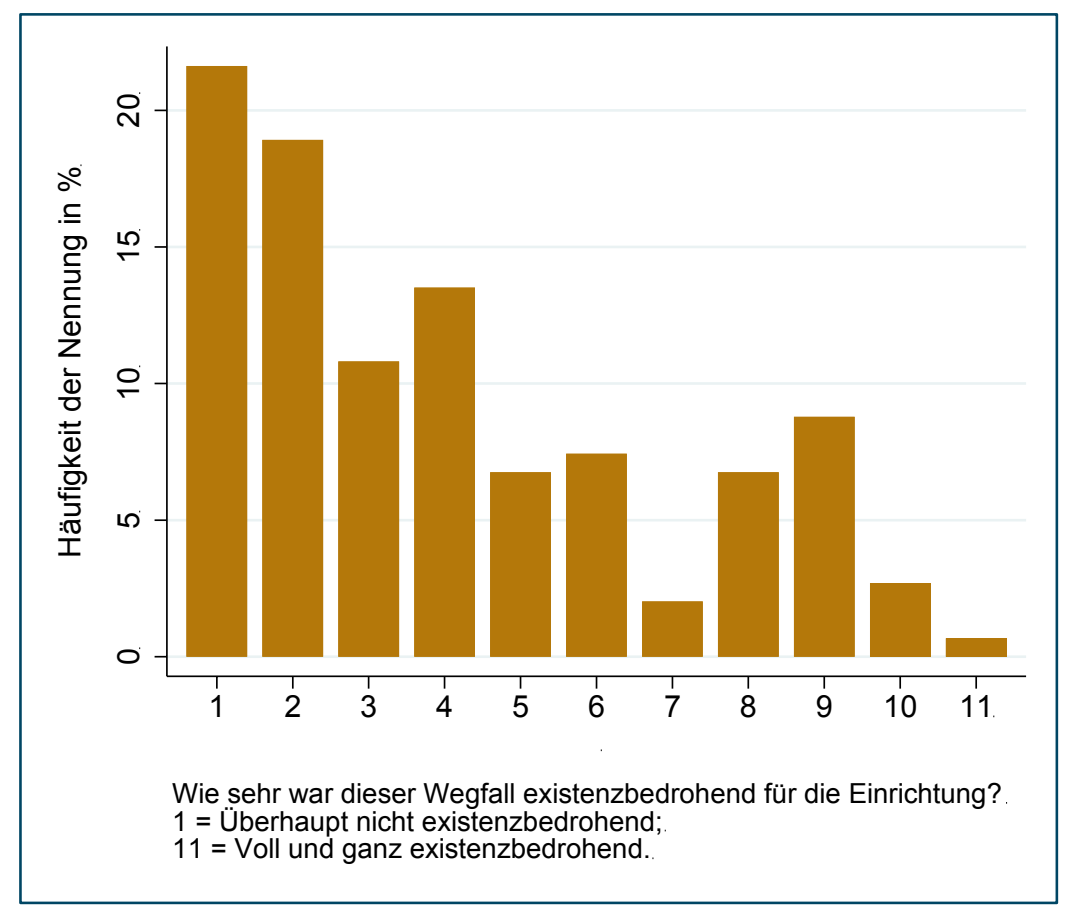

Quelle: ifb-Befragung „kontakt.los! 2020" ( $=402$; "trifft nicht $z u^{\prime \prime}=113$; keine Angabe = 141) 
Von diesen Einrichtungen, die eine Einschätzung vorgenommen haben, nimmt mehr als die Hälfte die Corona-Pandemie nicht oder kaum als existenzbedrohend wahr: $51 \%$ der Einrichtungen wählte den Wert 1 (also „überhaupt nicht existenzbedrohend“), 2 oder 3. Die andere Hälfte sieht hingegen durchaus eine Bedrohung, aber in sehr unterschiedlichem Ausmaß. Immerhin jede fünfte Einrichtung (20\%), die hier eine Einschätzung vorgenommen hat, sieht sich finanziell so sehr existenzbedroht, dass sie den Wertebereich der hinteren Kategorien (7 bis 11) wählte (vgl. Abbildung 4).

Erneut finden sich hier Unterschiede nach Einrichtungstyp: Während der Großteil der Familienstützpunkte (FSP) ihre Existenz nicht oder kaum bedroht sieht, bewerteten $58 \%$ der Familienbildungsstätten (FBS), die sich in stärkerem Ausmaß über Teilnahmegebühren finanzieren, ihre Existenz durch die Pandemie als sehr bedroht (Werte 7 oder höher). Mütter- und Familienzentren (MFZ) lassen sich dazwischen verorten: 54 \% bewerteten sich etwas existenzbedroht, $23 \%$ sehr existenzbedroht (vgl. Abbildung 5).

Abbildung 5: Existenzbedrohung der Familienbildungseinrichtungen

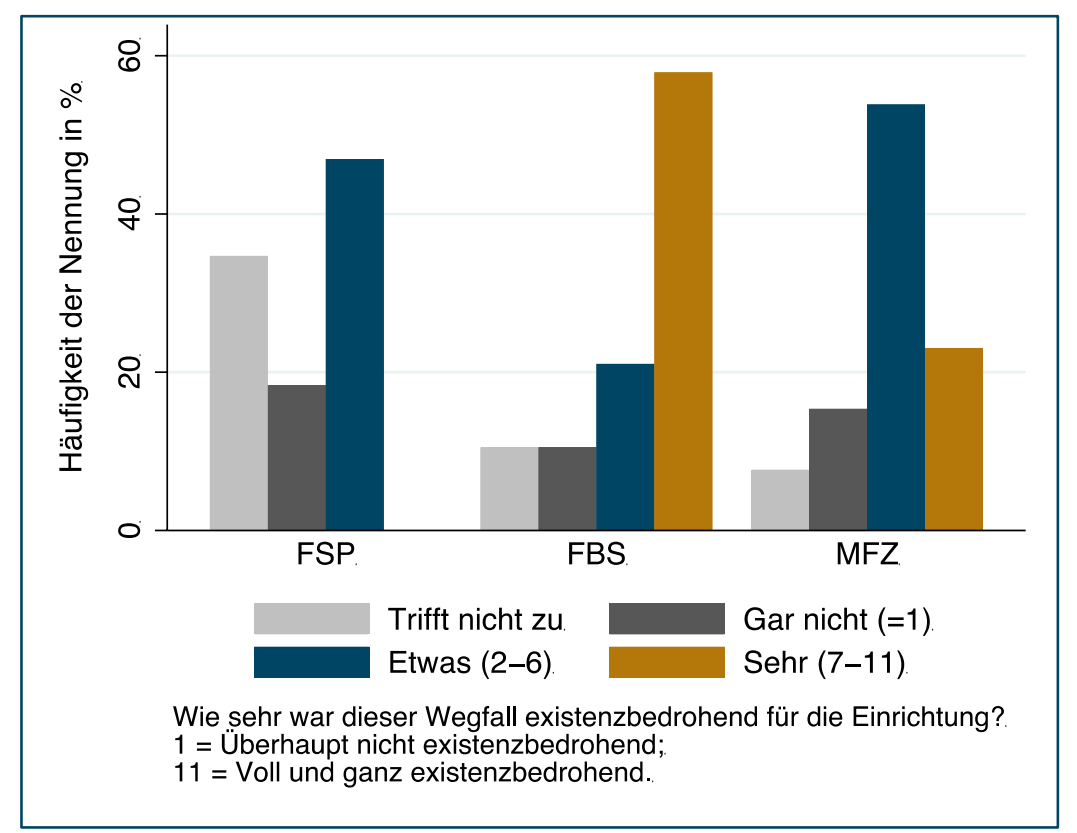

Quelle: ifb-Befragung „kontakt.los! 2020“ (Familienbildungseinrichtungen: $n=136$; keine Angabe = 42)

Von den Beratungsstellen sehen sich insbesondere die Ehe- und Familienberatungsstellen (EFB) durch Einnahmeausfälle etwas oder sehr bedroht (48 \% bzw. $29 \%$ ). Von den Erziehungsberatungsstellen (EB) sehen mehr als die Hälfte (54\%) eine gewisse Bedrohung, $4 \%$ sehen sich gar sehr bedroht; bei den Schwangerschaftsberatungsstellen (SB) liegen diese Werte bei 26 \% bzw. $11 \%$ (vgl. Abbildung 6). 
Abbildung 6: Existenzbedrohung der Beratungsstellen

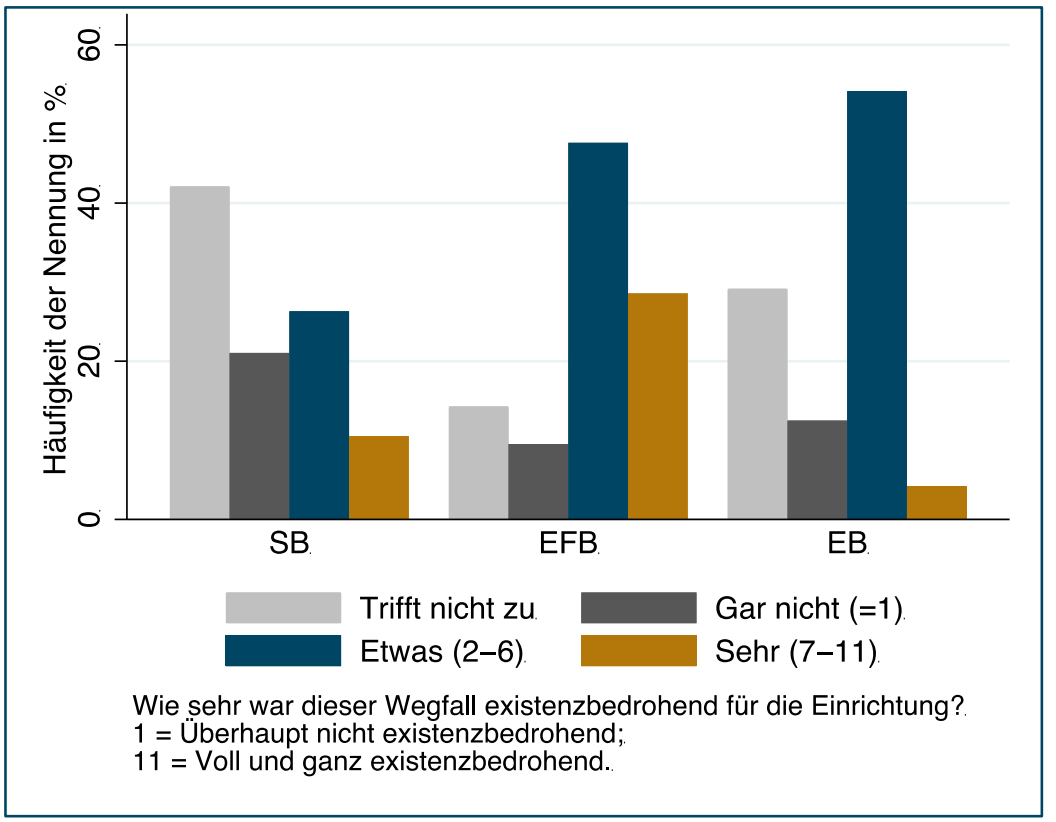

Quelle: ifb-Befragung „kontakt.los! 2020" (Beratungsstellen: $n=196$; keine Angabe = 94)

Staatliche Hilfsprogramme sollen die wirtschaftlichen Folgen der Corona-Pandemie abmildern. Einige Einrichtungen ( $8 \%$ ) nehmen diese Programme auch entsprechend wahr und erklärten, dass durch diese ein Großteil der Ausfälle kompensiert wird, während sie bei anderen Einrichtungen (11\%) nur einen kleinen Teil ersetzen können. Der Großteil der Einrichtungen (82 \%) kann zum Zeitpunkt der Befragung noch nicht abschätzen, welcher Anteil der Einnahmeausfälle durch staatliche Hilfsprogramme kompensiert werden wird (vgl. Abbildung 7).

Abbildung 7: Kompensation der Einnahmeausfälle durch staatliche Hilfsprogramme

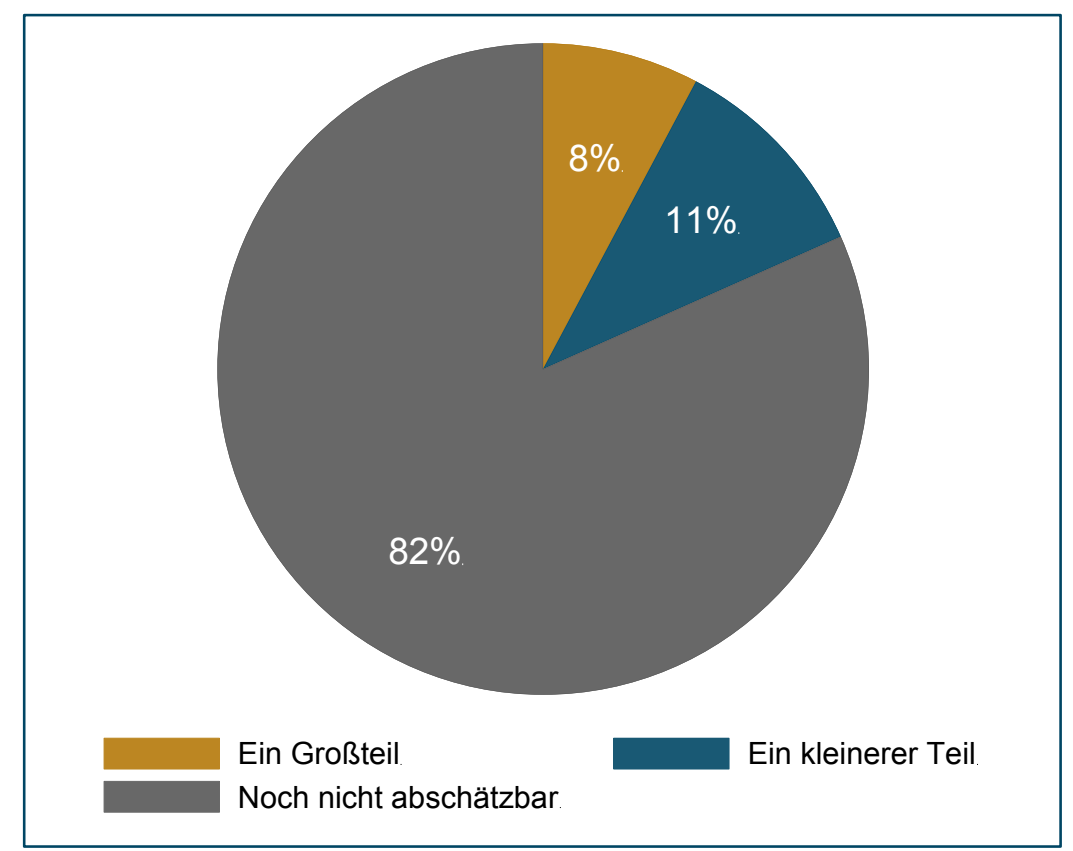

Quelle: ifb-Befragung „kontakt.los! 2020” ( $n=402 ;$ "trifft nicht $z u^{\prime \prime}=187$; keine Angabe = 111) 
Deutlich werden jedoch Unterschiede nach Einrichtungstyp: Keine der befragten Familienbildungsstätten (FBS) und keine der befragten Ehe- und Familienberatungsstellen (EFB) ist der Auffassung, dass die staatlichen Hilfspakete einen Großteil der Einnahmeausfälle kompensieren können. Stattdessen wird von je rund einem Fünftel vermutet, dass nur ein kleinerer Teil kompensiert werden kann; die anderen geben an, dies noch nicht abschätzen zu können. Die Familienstützunkte (FSP), die Mütter- und Familienzentren (MFZ), die Schwangerschafts- (SB) sowie die Erziehungsberatungen (EB) können die voraussichtliche Kompensation der Einnahmeausfälle größtenteils ebenfalls noch nicht abschätzen, sind aber verhalten optimistischer (vgl. Abbildung 8).

Abbildung 8: Kompensation der Einnahmeausfälle durch staatliche Hilfsprogramme

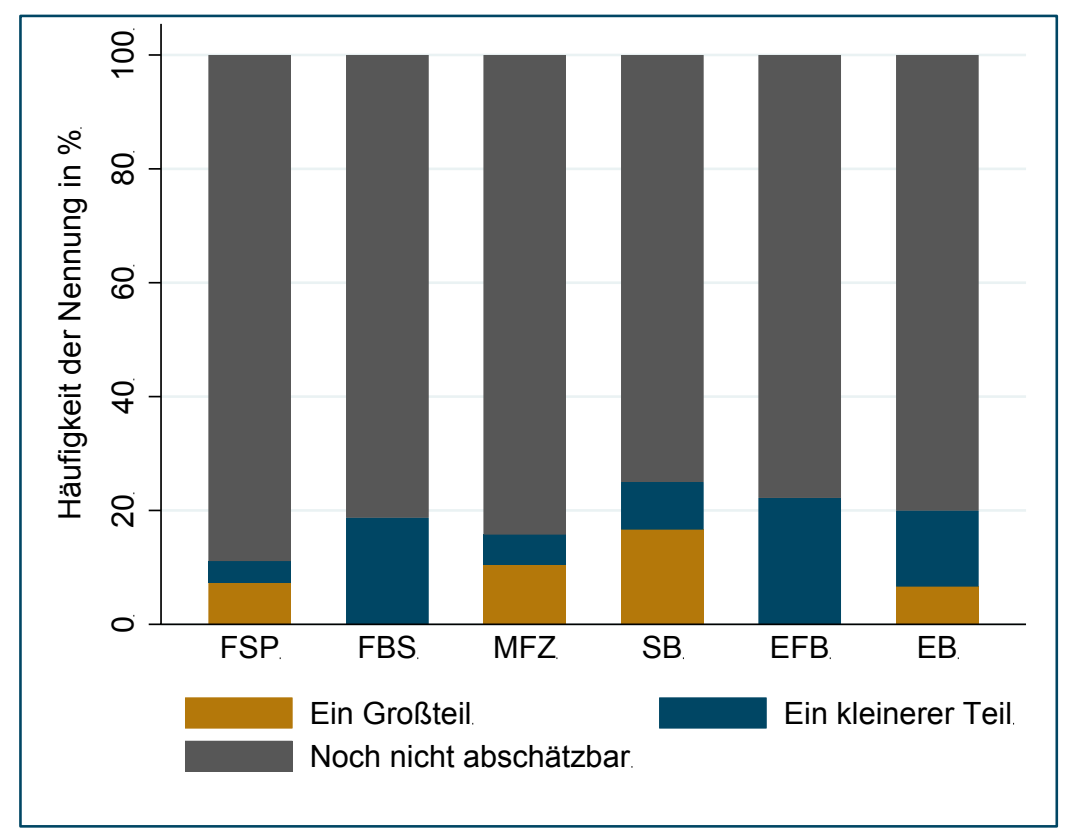

Quelle: ifb-Befragung „kontakt.los! 2020” ( $n=402$; "trifft nicht $z u^{\prime \prime}=187$; keine Angabe = 111)

Die Corona-Pandemie wirkt sich auch auf die Personalsituation in den Einrichtungen aus: auf die Situation der Angestellten und insbesondere auf die der freiberuflich tätigen Honorarkräfte - und somit auf die Zusammensetzung im Team allgemein. ${ }^{9}$

Von den Familienbildungseinrichtungen gaben $38 \%$ an, aufgrund der Pandemie die Weiterbeschäftigung von Honorarkräften ausgesetzt zu haben. Darüber hinaus haben $10 \%$ Neueinstellungen gestoppt oder bestehende Verträge auslaufen lassen. Auch die fest angestellten Mitarbeitenden spürten die Folgen: 15 \% der Einrichtungen im Bereich der Familienbildung beantragten Kurzarbeit für das Team oder Teile des Teams. Eine weitere Einschränkung ist, dass bei einem knappen Viertel der Familienbildungsinstitutionen Mitarbeitende die Arbeitszeit reduzieren mussten, um eigene Kinder zu betreuen; und ein knappes Fünftel gab an, dass Mitarbeitende nicht wie gewohnt arbeiten konnten, weil sie selbst zur Risikogruppe gehören. Nur ein gutes Drittel der Familienbildungseinrichtungen musste keine personellen Veränderungen vornehmen (vgl. Abbildung 9).

9 Zur Einschätzung der Veränderungen bezüglich der personellen Situation wurden die Befragten gebeten, aus mehreren Aussagen die zutreffenden anzukreuzen („Bitte wählen Sie aus, was auf Sie zutrifft: Aufgrund von Corona ..."); Mehrfachantworten waren möglich. 
Abbildung 9: Veränderungen personeller Ressourcen: Familienbildungseinrichtungen

\section{Aufgrund der Corona-Pandemie}

... haben wir die Weiterbeschäftigung von Honorarkräften ausgesetzt.

... haben wir Kurzarbeit beantragt.

... haben wir Neueinstellungen gestoppt oder bestehende Verträge auslaufen lassen.

... wurden MA*innen zeitweise in anderen Arbeitsbereichen des Trägers eingesetzt. ... haben MA*innen ihre Arbeitszeit reduziert, um eigene Kinder zu betreuen.

... können MA*innen nicht wie gewohnt arbeiten, da sie zur Risikogruppe gehören.

... haben wir personell keine Änderungen vorgenommen.

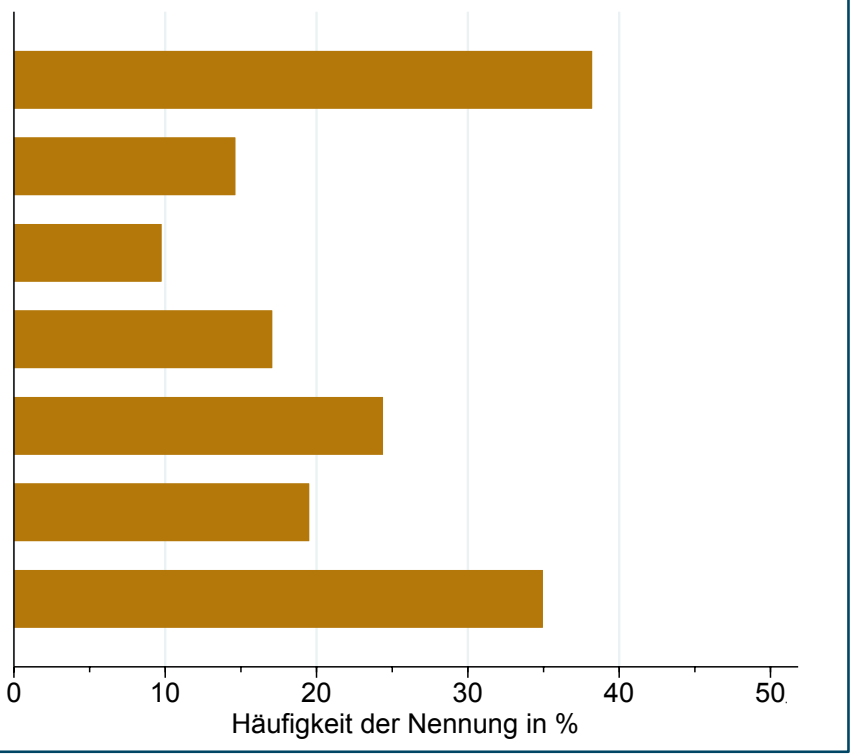

Quelle: ifb-Befragung „kontakt.los! 2020" (Familienbildungseinrichtungen: $n=137$; keine Angabe = 14) Anmerkung: Zustimmungswerte in \%, Mehrfachnennungen möglich

In den Beratungsstellen lassen sich ähnliche Tendenzen ausmachen, aber weit weniger drastische als bei den Familienbildungseinrichtungen: Keine Beratungsstelle gab an, Kurzarbeit beantragt zu haben und nur in wenigen Fällen musste man Verträge auslaufen lassen oder Neueinstellungen stoppen (4 \%) (vgl. Abbildung 10).

Abbildung 10: Veränderungen personeller Ressourcen: Beratungsstellen

\section{Aufgrund der Corona-Pandemie}

... haben wir die Weiterbeschäftigung von Honorarkräften ausgesetzt.

... haben wir Kurzarbeit beantragt.

... haben wir Neueinstellungen gestoppt oder bestehende Verträge auslaufen lassen.

... wurden MA*innen zeitweise in anderen Arbeitsbereichen des Trägers eingesetzt.

... haben MA*innen ihre Arbeitszeit reduziert, um eigene Kinder zu betreuen.

... können MA*innen nicht wie gewohnt arbeiten, da sie zur Risikogruppe gehören.

... haben wir personell keine Änderungen vorgenommen.

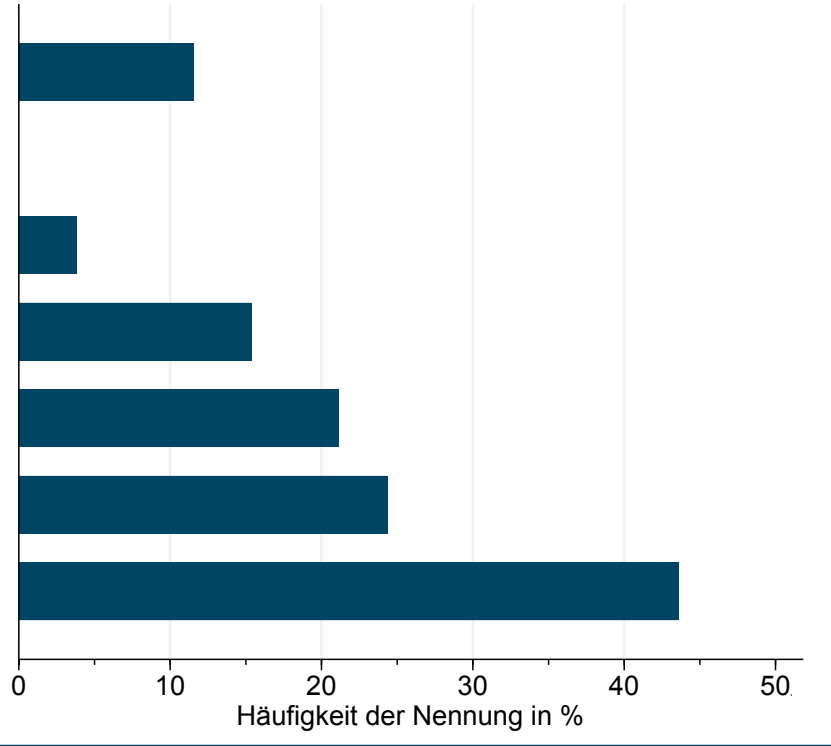

Quelle: ifb-Befragung „kontakt.los! 2020“ (Beratungsstellen: $n=203$; keine Angabe $=47$ ) 
Weil Honorarkräfte hier gemeinhin weniger eingesetzt werden, musste auch deren Weiterbeschäftigung nur bei einem kleinen Anteil ausgesetzt werden. Dennoch trifft dies auf $12 \%$ der Beratungsstellen zu. Weniger Unterschiede zu den Familienbildungseinrichtungen zeigen sich bezüglich der Arbeitszeitreduktion: Auch rund ein Fünftel der Beratungsstellen gab an, dass Mitarbeitende ihre Arbeitszeit reduzieren mussten, um eigene Kinder zu betreuen, und rund ein Viertel gab an, dass Mitarbeitende nicht wie gewohnt arbeiten konnten, weil sie selbst zur Risikogruppe gehören (vgl. Abbildung 10).

Auch in den KoKis wirkte sich die Pandemie auf die personellen Ressourcen aus: Einige wenige Stellen setzten die Beschäftigung von Honorarkräften aus (6\%), bei $11 \%$ wurden Mitarbeitende in andere Arbeitsbereiche versetzt, in $21 \%$ der KoKis mussten Teammitglieder ihre Arbeitszeit aufgrund von Kinderbetreuung reduzieren und in $17 \%$ konnten einige aufgrund der Zugehörigkeit zur Risikogruppe nicht wie gewohnt ihrer Arbeit nachgehen. Andererseits gab eine befragte KoKi sogar an, dass das Team aufgrund der Corona-Pandemie vergrößert wurde, und in knapp über der Hälfte der KoKis wurden personell keine Änderungen vorgenommen (vgl. Abbildung 11).

Abbildung 11: Veränderungen personeller Ressourcen: KoKis

\section{Aufgrund der Corona-Pandemie}

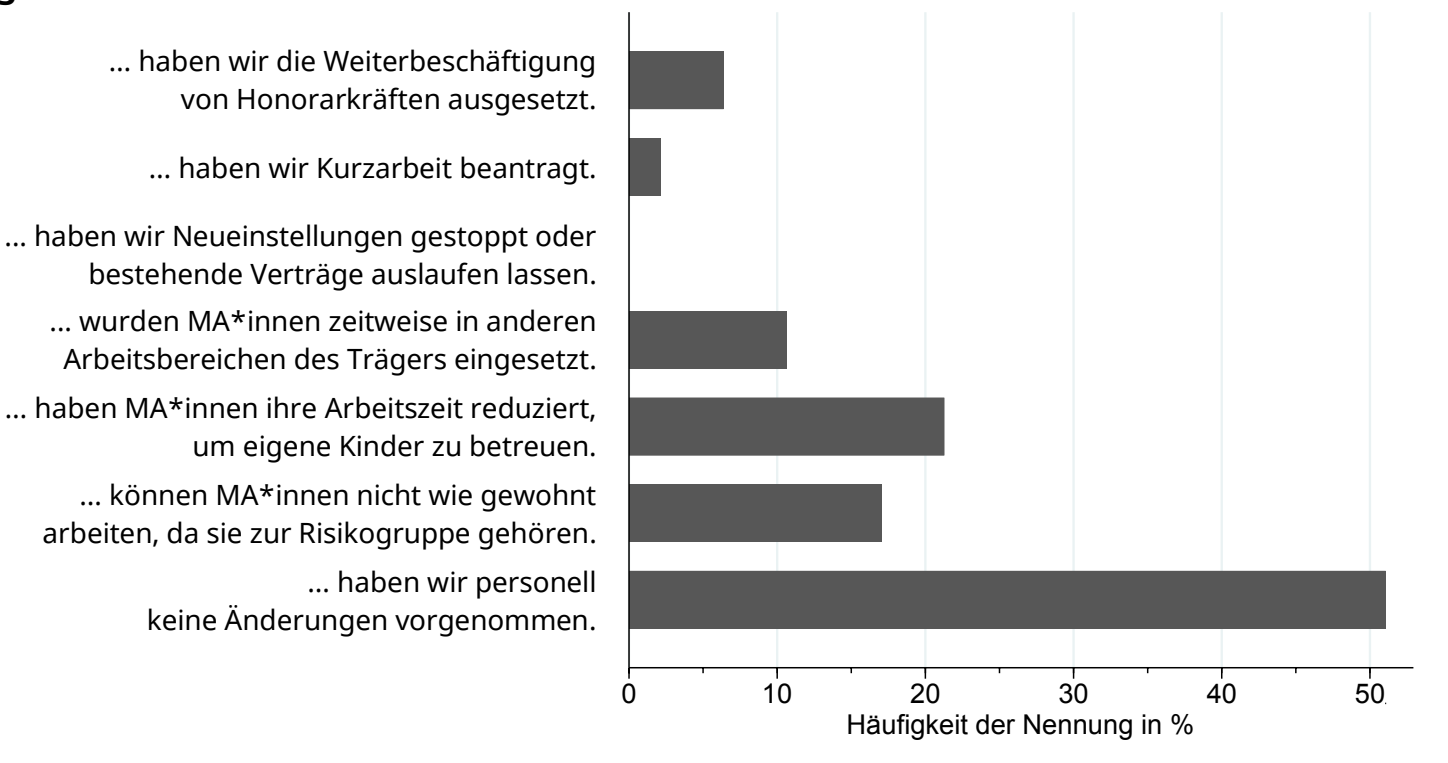

Quelle: ifb-Befragung „kontakt.los! 2020” (KoKis: $n=58$; keine Angabe =11)

Anmerkung: Zustimmungswerte in \%, Mehrfachnennungen möglich

Insgesamt fallen die personellen Auswirkungen in den KoKis somit am geringsten, bei den Familienbildungseinrichtungen am gravierendsten aus. Hierzu passend lassen sich zudem Unterschiede je nach Träger ausmachen: Einrichtungen in freier Trägerschaft mussten die Weiterbeschäftigung der Honorarkräfte besonders häufig aussetzen, häufiger Kurzarbeit beantragen und häufiger bestehende Arbeitsverträge auslaufen lassen. 


\section{Die Anliegen der Adressatinnen und Adressaten}

Mit der Corona-Pandemie haben sich massive Veränderungen im Alltag von Familien ergeben: (zeitweise) Schließungen von Bildungs- und Betreuungseinrichtungen und damit einhergehend Home-Schooling und Kinderbetreuung zu Hause, Arbeiten im HomeOffice (soweit möglich), Kontaktbeschränkungen und Abstandsregeln sowie Einschränkungen von Sport-, Kultur- und Freizeitangeboten. All dies bedeutet, dass das Familienleben reorganisiert werden musste - und immer noch muss. Stehen Familien vor Veränderungen und neuen Herausforderungen, ist der Bedarf an Orientierung und Unterstützung gewöhnlich besonders hoch.

Um die Veränderungen der Bedarfe von Familien während dieser besonderen Situation abzuschätzen, wurden die Expertinnen und Experten gefragt, mit welchen Themen sich Eltern und Schwangere üblicherweise an die Fachpraxis wenden (Referenzjahr: 2019) und welche Anliegen während der Corona-Pandemie im Mai 2020 an sie herangetragen wurden. ${ }^{10}$

Allen Einrichtungstypen gemeinsam ist, dass sich Familien zuletzt besonders häufig und häufiger als vor der Pandemie - mit Krisen, Problemlagen, belastenden Ereignissen an die Fachkräfte gewandt haben. Zu den fünf meistgenannten Themen zählen bei allen Einrichtungsgruppen des Weiteren die kindliche Entwicklung und Erziehung sowie psychische Gesundheit, Ängste und Mobbing, und damit ein Themenbereich, der insbesondere bei den familienbildenden Einrichtungen nicht zu den Kernthemen zählt.

Abbildung 12: Anliegen der Eltern/Schwangeren in Beratungsstellen während der Corona-Pandemie im Mai 2020

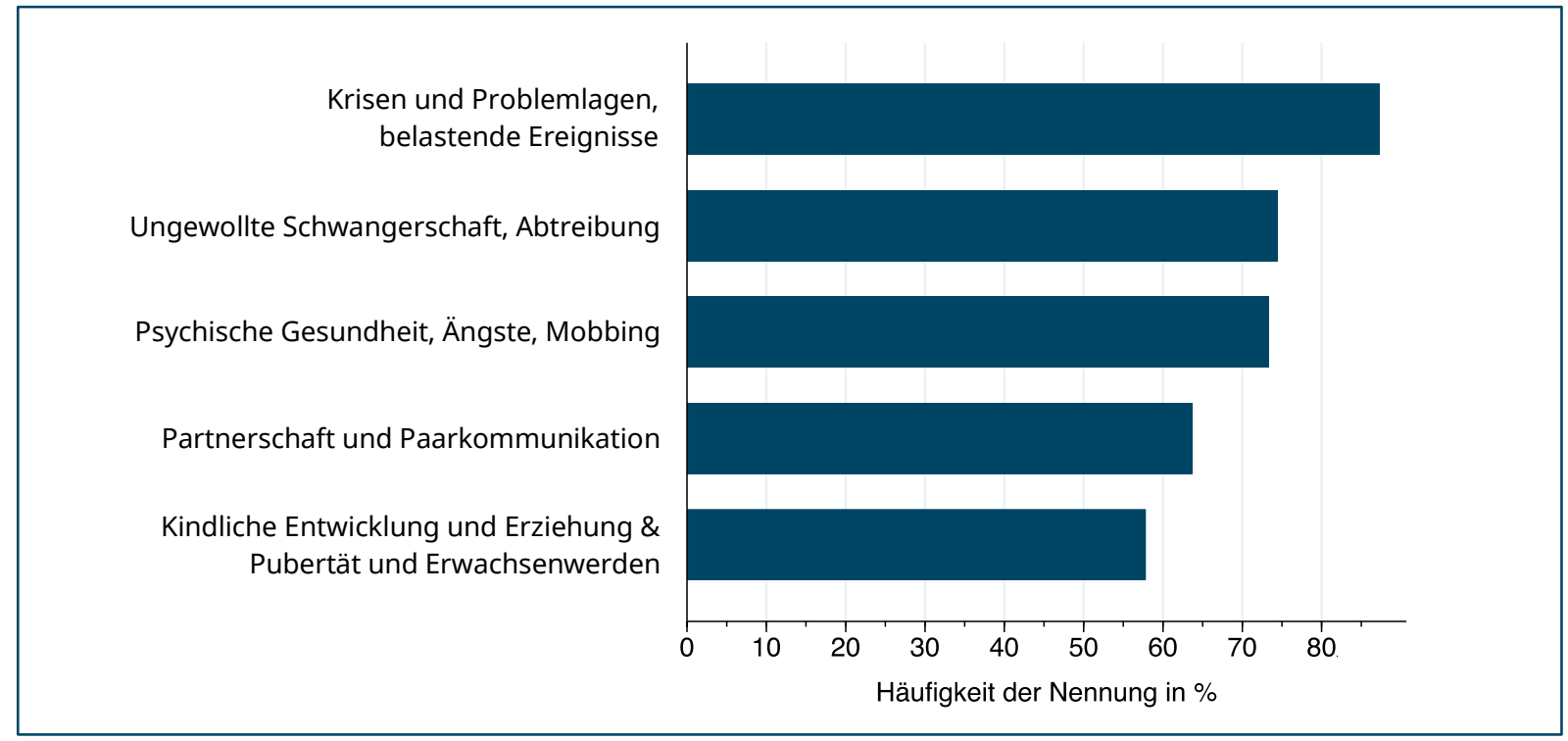

Quelle: ifb-Befragung „kontakt.los! 2020" (Beratungsstellen: $n=203$; keine Angabe = 38)

Anmerkung: Mehrfachnennungen möglich

10 Die Themenliste wurde vorgegeben und variierte leicht nach Einrichtungstyp, um die jeweils typischen Themen für die Einrichtungen erfassen zu können. Mehrfachnennungen waren möglich. 
Gerade bei Beratungsstellen nehmen die beiden Themenschwerpunkte Krisen und Problemlagen, belastende Ereignisse (87\%) und psychische Gesundheit (73\%) einen wichtigen Stellenwert ein; weiterhin stehen die inhaltlichen Schwerpunkte der jeweiligen Beratungsstellen ganz oben: ungewollte Schwangerschaft/Abtreibung (74\%), Paarkommunikation und Partnerschaft (64\%) sowie kindliche Entwicklung und Erziehung und Pubertät und Erwachsenwerden (je 58 \%) (vgl. Abbildung 12).

Die Koordinierenden Kinderschutzstellen KoKis sind in Jugendämtern angesiedelt und haben die Aufgabe, Eltern mit Kindern im Alter von 0 bis 3 Jahren in belastenden Lebenslagen und Überforderungssituationen kostenfrei zu beraten und zu unterstützen. Dementsprechend bewegen sich die Themen der Eltern, die sich an KoKis wenden, im typischen Spektrum der Frühen Hilfen und wichtige Anliegen sind naturgemäß Schwangerschaft, Geburt und erstes Lebensjahr (86\%) sowie kindliche Entwicklung und Erziehung (68\%). Zu den häufigsten Anliegen gehören aber auch hier Krisen und Problemlagen, belastende Ereignisse (90\%), psychische Gesundheit, Ängste und Mobbing (80 \%) sowie Fragen zur institutionellen Betreuung der unter 6-Jährigen (Krippe, Kindergarten, Kindertagespflege, also beispielsweise einen Betreuungsplatz finden, Fragen der Eingewöhnung; $66 \%$ ) (vgl. Abbildung 13). Und während die drei letztgenannten Aspekte im Zuge der Pandemie an Bedeutung gewonnen haben, sind z. B. Anliegen zu Haushaltsführung und Behördliches weiter in den Hintergrund gerückt.

Abbildung 13: Anliegen der Eltern/Schwangeren in KoKis

während der Corona-Pandemie im Mai 2020

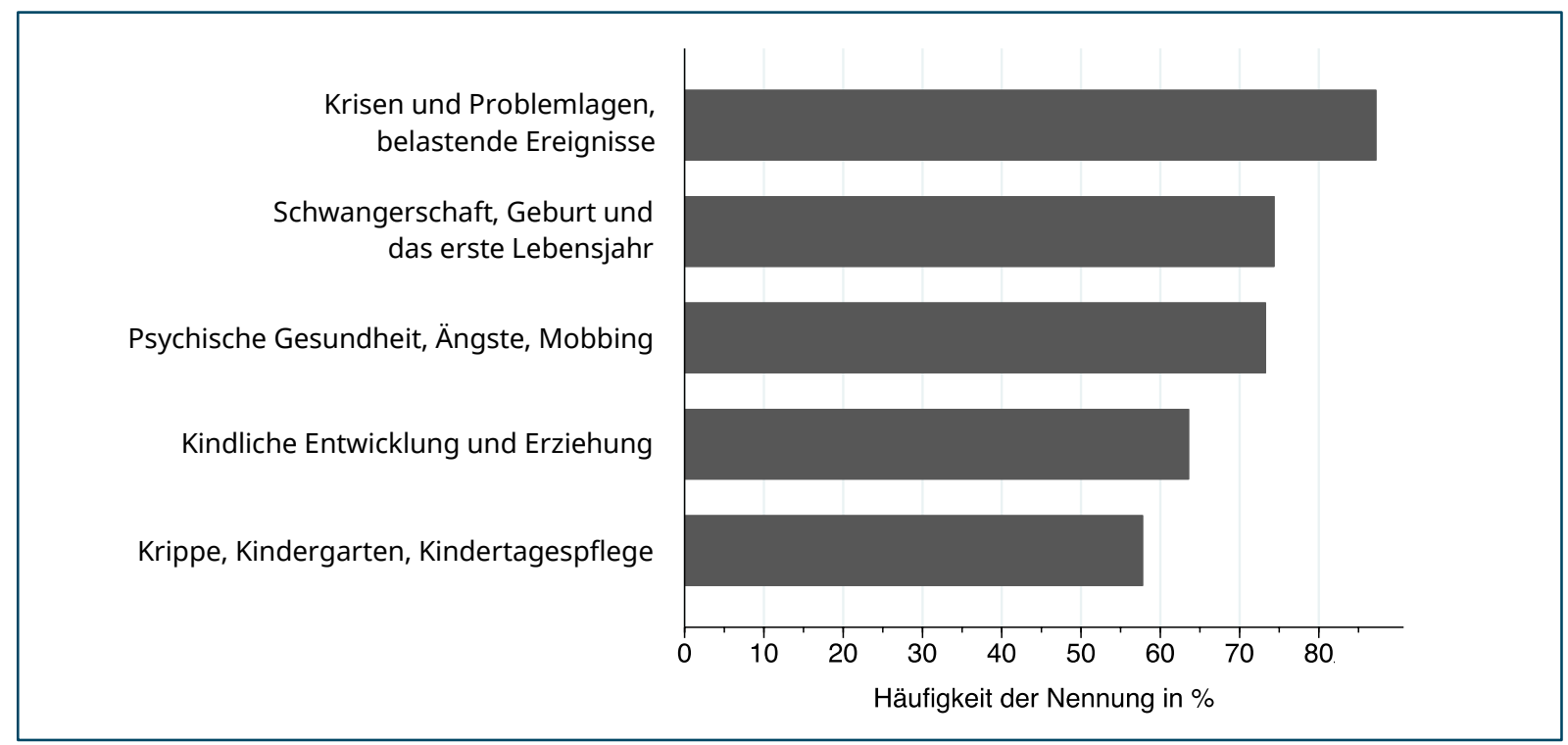

Quelle: ifb-Befragung „kontakt.los! 2020” (KoKis: $n=58$; keine Angabe = 8)

Anmerkung: Mehrfachnennungen möglich

Das zentrale Thema der Familienbildungseinrichtungen, kindliche Entwicklung und Erziehung, ist auch während der Pandemie von großer Bedeutung (65 \% der Fachkräfte nennen dies als häufiges Anliegen), jedoch wurden andere Themen dringlicher für die Familien: Neben den bereits genannten Krisen, Problemlagen und belastenden Ereignissen (74 \%) wurden besonders häufig Anliegen rund um die institutionelle Betreuung (Krippe, Kindergarten und Kindertagespflege; 72 \%) sowie um die Vereinbarkeit von Familie und Beruf 
(61\%) an die Fachkräfte herangetragen; auch das Thema der psychischen Gesundheit gewinnt an Bedeutung (50 \%) (vgl. Abbildung 14).

Abbildung 14: Anliegen der Eltern/Schwangeren in Familienbildungseinrichtungen während der Corona-Pandemie im Mai 2020

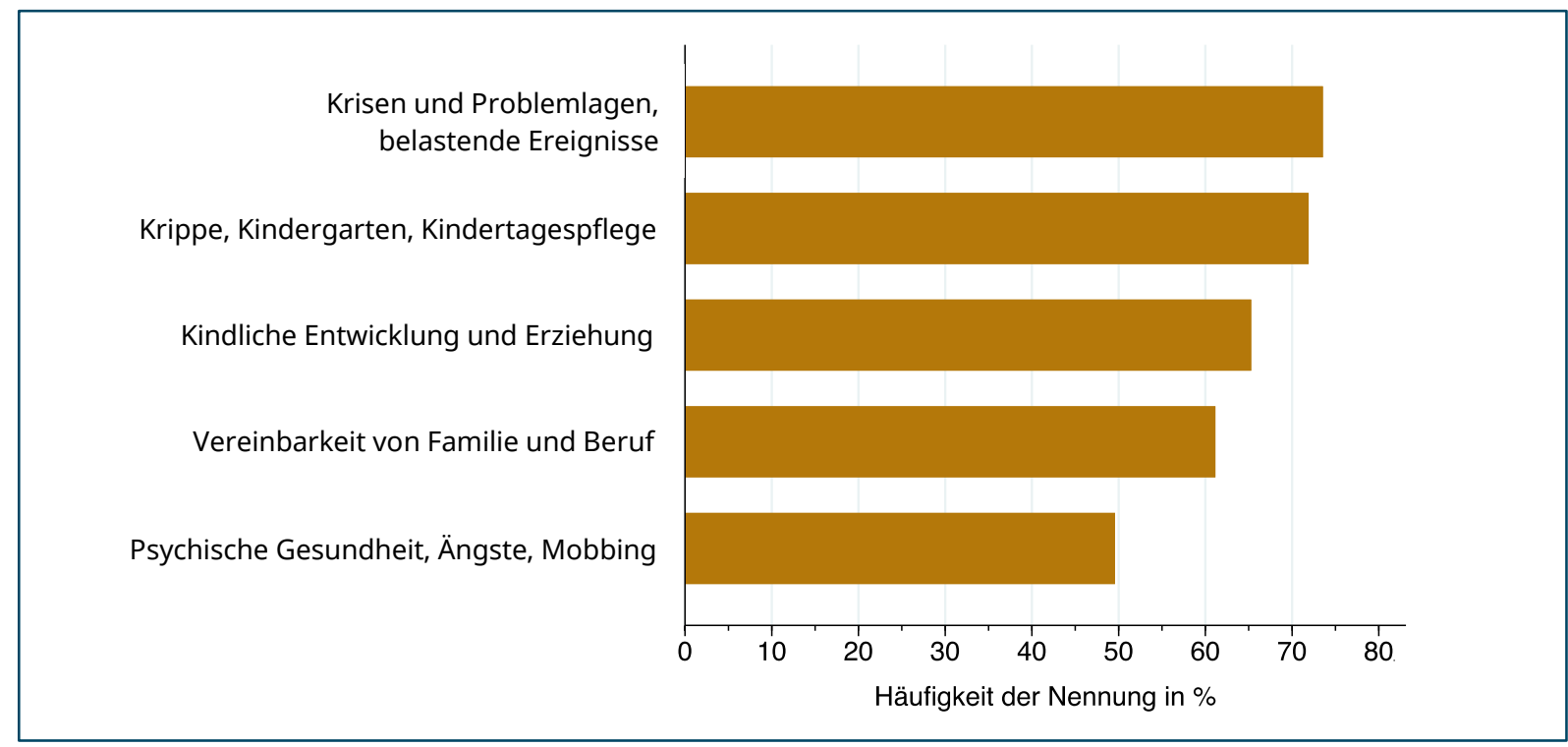

Quelle: ifb-Befragung „kontakt.los! 2020" (Familienbildungseinrichtungen: $n=137$; keine Angabe = 16) Anmerkung: Mehrfachnennungen möglich

Im Vergleich zu den Anliegen, mit denen die Adressatinnen und Adressaten noch im Jahr 2019 an die Fachpraxis herantraten, hat sich die Rangfolge der häufigsten Themen also mit Ausbruch der Corona-Pandemie in allen drei Arbeitsbereichen verschoben (vgl. Abbildung 15).

Ganz besonders deutlich wird dies bei den Einrichtungen der Familienbildung. Bei Elterngesprächen sind die Themenbereiche psychische Gesundheit, Ängste, Mobbing und Vereinbarkeit von Familie und Beruf wie auch Krisen und Problemlagen, belastende Ereignisse jeweils mehrere Ränge nach oben gerutscht und verdeutlichen Veränderungen in den Bedarfen von Eltern. Dagegen haben Fragen rund um Freizeitgestaltung, Kreativität und Musik (derzeit Rang 9) deutlich an Gewicht verloren. Der Eindruck einer Fachkraft, dass derzeit gerade junge Familien schlechter erreicht werden, bestätigt sich auch beim Thema Schwangerschaft, Geburt und das erste Lebensjahr, das von Rang 3 auf Rang 8 verdrängt wurde.

Diese Entwicklung in Familienbildungseinrichtungen, Beratungsstellen und bei KoKis zeigt, dass Schwangere und Eltern, die sich an die Fachpraxis wenden, dies überdurchschnittlich oft aufgrund von Sorgen, Belastungen und Herausforderungen tun. Diese sind mit Sicherheit im Zusammenhang mit der angespannten Vereinbarkeitsthematik und der Betreuungssituation zu sehen, was sich auch in den offenen Angaben zeigt, in denen die Fachkräfte darauf hinweisen, dass sich Eltern „einfach entlasten und wieder Zuversicht finden [möchten] durch ein Gespräch". Eine Fachkraft merkt an, dass Eltern "nicht mit der Erziehung, sondern mit dem Home-Schooling überfordert"seien, eine andere betont die "Überforderung durch Betreuung der Kinder und Home-Office". Auffallend wenige Fachkräfte merken auch positive Aspekte an, wenn sie über ihre Erfahrungen berichten: etwa, 
dass sich aufgrund der Corona-Pandemie "mehr Väter melden", "Eltern ihre Kinder genießen" und es auch Familien gab, "bei denen sich sogar Probleme durch mehr gemeinsame Zeit reduziert haben".

Abbildung 15: Rangfolge der am häufigsten genannten Themen

\begin{tabular}{|c|c|c|c|c|c|}
\hline \multicolumn{2}{|r|}{$\begin{array}{c}\text { Einrichtungen } \\
\text { der Familienbildung }\end{array}$} & \multicolumn{2}{|r|}{ Beratungsstellen } & \multicolumn{2}{|r|}{ KoKis } \\
\hline $\begin{array}{l}1 . \\
\text { 5. }\end{array}$ & $\begin{array}{l}\text { Krisen \& Problemlagen, } \\
\text { belastende Ereignisse }\end{array}$ & $\begin{array}{l}1 . \\
1 .\end{array}$ & $\begin{array}{l}\text { Krisen \& Problemlagen, } \\
\text { belastende Ereignisse }\end{array}$ & $\begin{array}{l}1 . \\
2 . \uparrow\end{array}$ & $\begin{array}{l}\text { Krisen \& Problemlagen, } \\
\text { belastende Ereignisse }\end{array}$ \\
\hline $\begin{array}{l}2 . \\
2 .\end{array}$ & $\begin{array}{l}\text { Krippe, Kindergarten, } \\
\text { Kindertagespflege }\end{array}$ & $\begin{array}{l}2 . \\
2 .\end{array}$ & $\begin{array}{l}\text { Ungewollte Schwanger- } \\
\text { schaft, Abtreibung }\end{array}$ & $\begin{array}{l}2 . \\
1 . \Downarrow\end{array}$ & $\begin{array}{l}\text { Schwangerschaft, } \\
\text { Geburt \& das erste } \\
\text { Lebensjahr }\end{array}$ \\
\hline $\begin{array}{l}3 . \\
1 . v\end{array}$ & $\begin{array}{l}\text { Kindliche Entwicklung } \\
\text { \& Erziehung }\end{array}$ & $\begin{array}{l}3 . \\
4 .\end{array}$ & $\begin{array}{l}\text { Psychische Gesundheit, } \\
\text { Ängste, Mobbing }\end{array}$ & $\begin{array}{l}3 . \\
5 . \uparrow\end{array}$ & $\begin{array}{l}\text { Psychische Gesundheit, } \\
\text { Ängste, Mobbing }\end{array}$ \\
\hline $\begin{array}{l}4 . \\
\text { 8. }\end{array}$ & $\begin{array}{l}\text { Vereinbarkeit von } \\
\text { Familie \& Beruf }\end{array}$ & $\begin{array}{l}4 . \\
\text { 3. } \downarrow\end{array}$ & $\begin{array}{l}\text { Partnerschaft \& } \\
\text { Paarkommunikation }\end{array}$ & $\begin{array}{l}4 . \\
3 . \Downarrow\end{array}$ & $\begin{array}{l}\text { Kindliche Entwicklung } \\
\text { \& Erziehung }\end{array}$ \\
\hline $\begin{array}{l}5 . \\
15 \uparrow\end{array}$ & $\begin{array}{l}\text { Psychische Gesundheit, } \\
\text { Ängste, Mobbing }\end{array}$ & $\begin{array}{l}5 . \\
6 . / 7 . \Downarrow\end{array}$ & $\begin{array}{l}\text { Kindliche Entwicklung \& } \\
\text { Erziehung/ } \\
\text { Pubertät \& Erwachsen- } \\
\text { werden }\end{array}$ & $\begin{array}{l}5 . \\
6 .\end{array}$ & $\begin{array}{l}\text { Krippe, Kindergarten, } \\
\text { Kindertagespflege }\end{array}$ \\
\hline $\boldsymbol{\uparrow}$ & \multicolumn{5}{|c|}{ Themenbereich hat im Mai vergleichsweise an Bedeutung gewonnen } \\
\hline$\bullet$ & \multicolumn{5}{|c|}{ Keine Veränderung der Rangfolge des Themenbereichs } \\
\hline 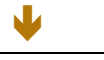 & \multicolumn{5}{|c|}{ Themenbereich hat im Mai vergleichsweise an Bedeutung verloren } \\
\hline
\end{tabular}

Quelle: ifb-Befragung „kontakt.los! 2020" ( $n=402$, keine Angabe =64)

Dass sich vor allem bei Familienbildungseinrichtungen Themen verschoben haben, lässt sich wohl mit ihrem sehr breiten Auftrag im Sinne einer (primär-)präventiven allgemeinen Förderung der Erziehung in der Familie erklären, zudem mit dem Selbstverständnis der Einrichtungen, als niedrigschwellige Anlaufstellen offen für alle Anliegen aller Familien zu sein. Im Kontext des gesellschaftlichen Wandels liegt das Potenzial der institutionellen Familienbildung darin, als „Seismograph“ schnell auf sich verändernde Bedarfe zu reagieren. Umso wichtiger ist auch ihre Einbindung in die Netzwerke Früher Hilfen und die Zusammenarbeit mit anderen Akteuren der Jugendhilfe, des Gesundheits- und Bildungsbereichs sowie ihre Lotsenfunktion zu weiterführenden Hilfen und Angeboten. Hier können Beratungsstellen mit ihrem spezifischen Kompetenzprofil individuelle Unterstützung leisten. 


\section{Die Corona-Pandemie als Digitalisierungsmotor?}

Aufgrund der Abstands- und Hygienemaßnahmen war und ist die Durchführung zahlreicher Präsenzveranstaltungen während der Corona-Pandemie nicht oder nur eingeschränkt möglich. Wie auch in vielen anderen gesellschaftlichen Bereichen stellt sich auch in der Familienbildung, der Beratung und in den Frühen Hilfen die Frage, inwiefern digitale Angebote gewinnbringend realisiert werden können und die Aussetzung von Präsenzangeboten kompensiert werden kann.

\section{Digitale Formate: Während und vor der Pandemie}

Im Zuge der Corona-Pandemie hat es einen Digitalisierungsschub gegeben: Im Mai 2020 haben Einrichtungen der Familienbildung und Beratung digitale Angebote insgesamt intensiver genutzt als früher. Um das Ausmaß und die Veränderungen der Digitalisierung zu erfassen, wurden die Einrichtungen gefragt, ob sie die nachfolgend dargestellten digitalen Angebote im Mai 2020 bereitgestellt haben, und wenn ja, wie häufig. Bei denjenigen, die ein digitales Angebot "nie“ angeboten haben, wurde davon ausgegangen, dass sie es auch vor der Pandemie nicht angeboten haben; bei denjenigen, die ein bestimmtes Format im Mai „selten“ oder häufiger angeboten haben, wurde nachgefragt, wie häufig jenes bereits vor der Corona-Pandemie, im Jahr 2019, angeboten wurde.

Das Kommunikationsmittel schlechthin ist das Telefon. 95 \% der Einrichtungen gaben an, im Jahr 2019 telefonisch beraten zu haben; im Mai 2020 stieg dieser Anteil auf $99 \%$. Und der Anteil derjenigen, die mehrmals pro Woche per Telefon beraten, fällt im Mai mit $81 \%$ deutlich höher aus als vor der Pandemie (47 \%) - die Nutzung von Telefonberatung/Hotlines hat sich also deutlich intensiviert (vgl. Abbildung 16).

\section{Abbildung 16: Digitale Angebote: Telefonische Beratung/Hotline}

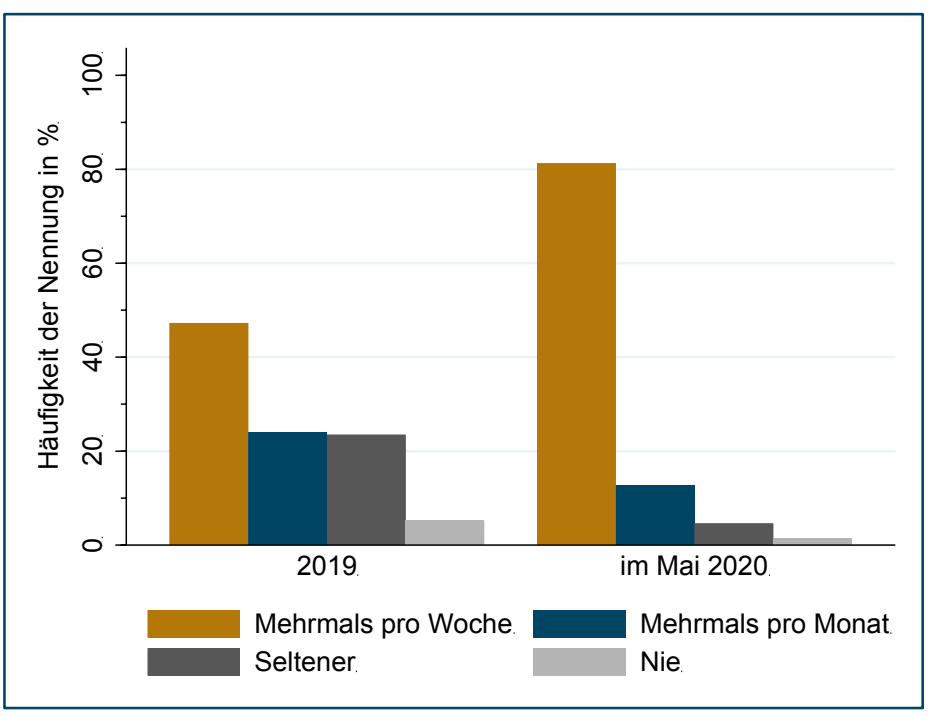

Quelle: ifb-Befragung „kontakt.los! 2020" ( $=402$; keine Angabe: "2019” = 53; "im Mai 2020" = 33)

Auch Beratung per E-Mail zählt zu digitalen Angeboten, welche die familienunterstützenden Einrichtungen bereits vor der Pandemie angeboten und während der Pandemie deutlich ausgebaut haben. Beinahe drei Viertel der befragten Institutionen boten 
mehrmals im Monat Mai, zum Teil mehrmals pro Woche, Beratung per E-Mail an. Vor der Pandemie lag dieser Anteil mit $56 \%$ deutlich niedriger. Auch der Anteil der Einrichtungen, die keine E-Mail-Beratung anboten, ist mit $8 \%$ im Mai deutlich geringer als vor der Pandemie (14\%) (vgl. Abbildung 17).

Abbildung 17: Digitale Angebote: Beratung per E-Mail

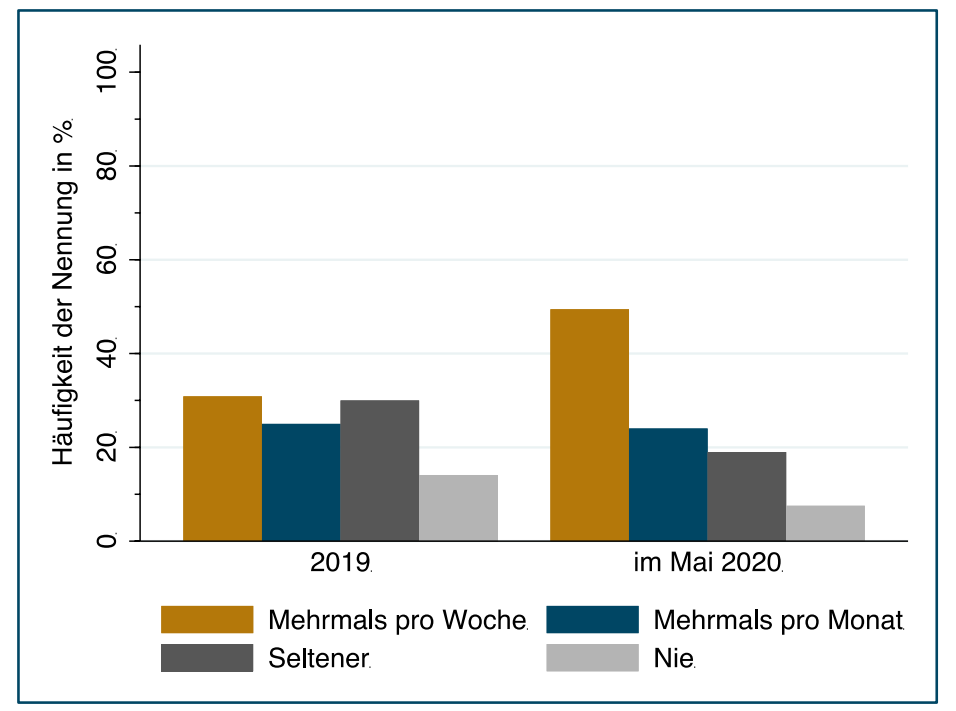

Quelle: ifb-Befragung "kontakt.los! 2020" ( $=$ 402; keine Angabe: "2019“ = 62; „im Mai 2020" = 44)

Ein weiteres Format, das rund zwei Drittel (68\%) der Einrichtungen im Mai nutzten, um in Kontakt mit ihrer Zielgruppe zu bleiben und diese zu informieren, waren Rundmailings. 56 \% der Einrichtungen nutzten diese bereits vor der Pandemie, um ihre Zielgruppen zu informieren, wobei ein Großteil diese eher sparsam einsetzte. Der Anteil derjenigen, die Rundmailings mindestens mehrmals im Monat nutzten, verdoppelte sich von $21 \%$ auf $43 \%$ (vgl. Abbildung 18).

Abbildung 18: Digitale Angebote: Rundmailings

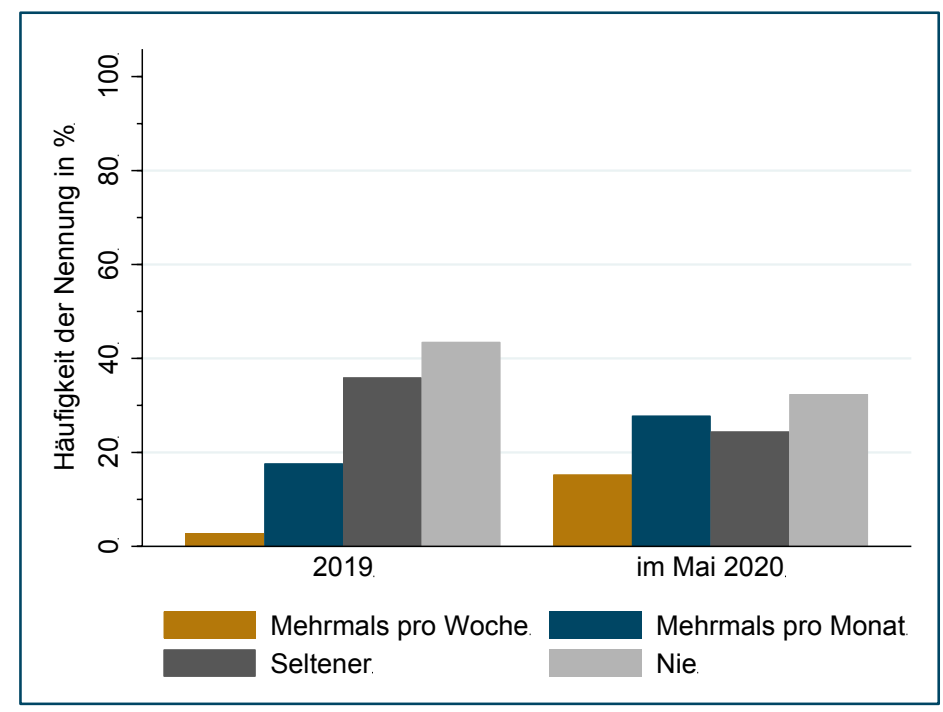

Quelle: ifb-Befragung „kontakt.los! 2020” ( $=$ 402; keine Angabe: "2019“ = 85; "im Mai 2020“ = 75) 
Über die Hälfte (55 \%) der befragten Anlaufstellen für Familien haben im Mai 2020 Beratungs- und Bildungsangebote per Video-Telefonie oder Video-Chat durchgeführt: $20 \%$ mehrmals pro Woche, $19 \%$ mehrmals im Monat und $16 \%$ seltener. Vor der Pandemie gehörte dies lediglich bei $5 \%$ der befragten Institutionen zum Spektrum. Damit zählt die Video-Telefonie/der Video-Chat zu den digitalen Formaten, die am stärksten ausgebauten wurden (vgl. Abbildung 19).

Abbildung 19: Digitale Angebote: Video-Telefonie/Video-Chat

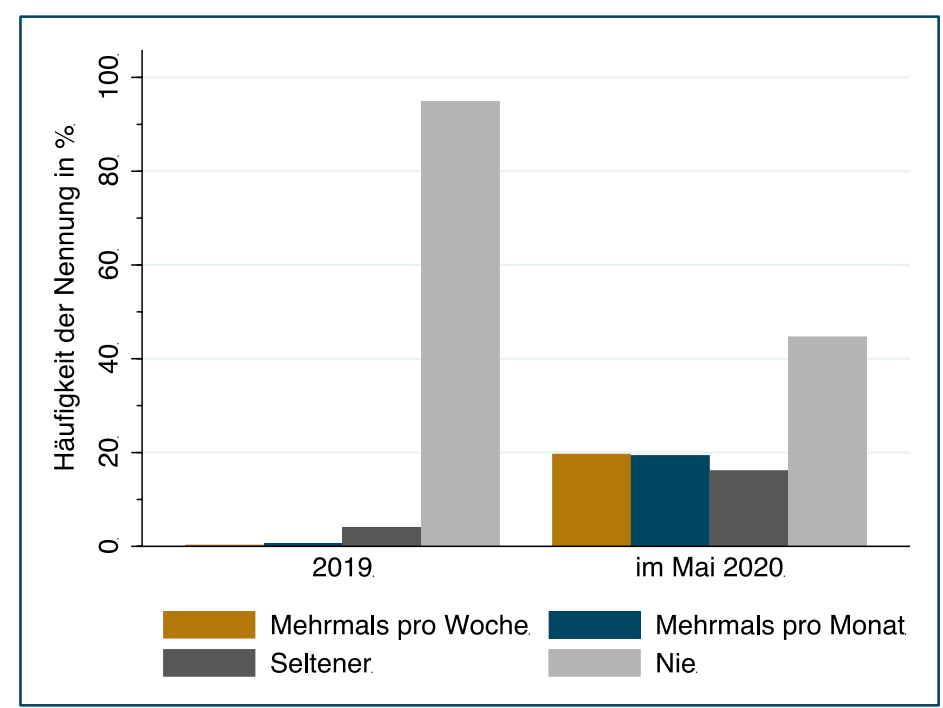

Quelle: ifb-Befragung „kontakt.los! 2020" ( $n=402$; keine Angabe: "2019” = 86; "im Mai 2020" =62)

Auch Gruppenchats und Messenger-Broadcast-Listen wurden im Mai 2020 von mehr Einrichtungen und von diesen auch häufiger genutzt als vor der Pandemie. $35 \%$ der Befragten gaben an, diese zu nutzen, um mit Familien in Kontakt zu bleiben; 20 \% nutzten dieses Medium bereits vor der Pandemie. Im Vergleich zu anderen digitalen Formaten kommen Chats und Listen seltener zum Einsatz, wurden im Mai aber immerhin bei $14 \%$ mehrmals pro Woche und bei $9 \%$ mehrmals pro Monat verwendet (vgl. Abbildung 20).

Abbildung 20: Digitale Angebote: Messenger-Broadcast-Liste/Gruppenchat

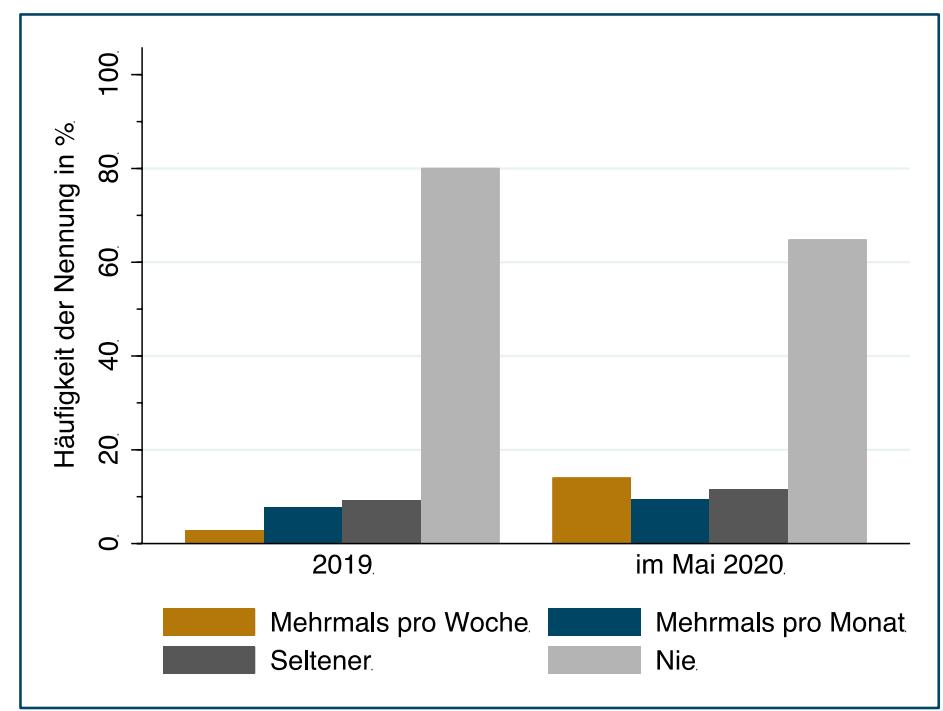

Quelle: ifb-Befragung „kontakt.los! 2020" ( $=402$; keine Angabe: "2019” = 90; "im Mai 2020" = 83) 
Klassische Präsenz-Angebote können digitalisiert werden, indem sie als Webseminare angeboten werden. Dies erfolgte jedoch nur sehr selten: Im Jahr 2019 haben lediglich $3 \%$ der Einrichtungen Webseminare umgesetzt. Für den Großteil der Einrichtungen waren Webseminare also ein Novum - aber immerhin ein Viertel der Einrichtungen probierte sich hieran aus und bot im Mai 2020 erstmalig Webseminare an. Konzipiert wurden sowohl Online-Elternkurse und -gruppen als auch Eltern-Kind-Gruppen, beispielsweise PEKiP online oder Online-Babymassage (vgl. Abbildung 21).

Abbildung 21: Digitale Angebote: Webseminare

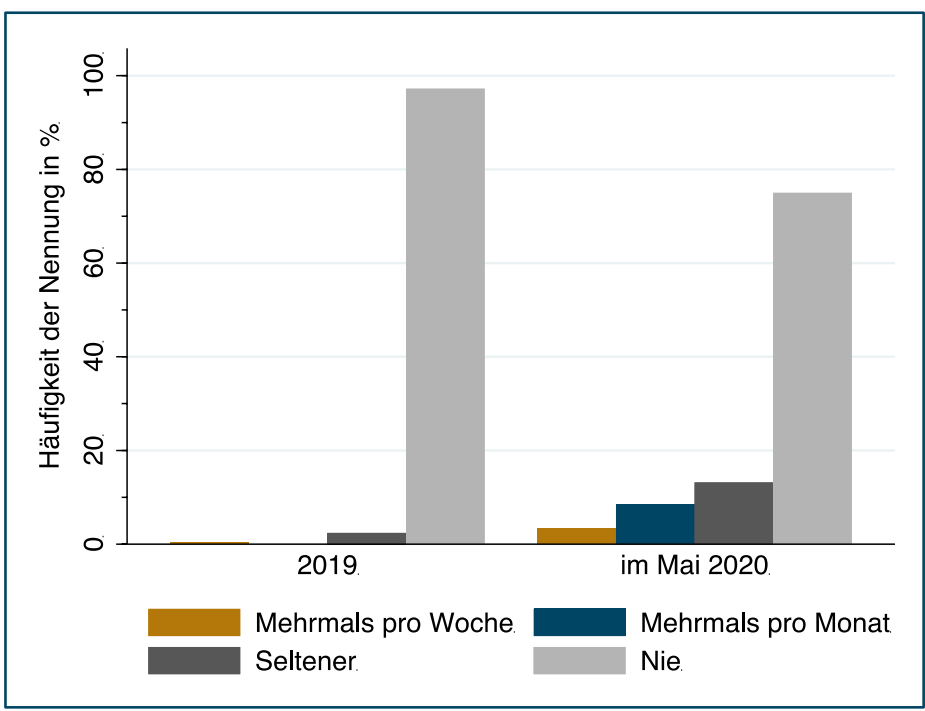

Quelle: ifb-Befragung "kontakt.los! 2020" ( $=$ 402; keine Angabe: "2019" = 113; "im Mai 2020" = 106)

Bereits vor der Pandemie war ein knappes Viertel (23\%) der Einrichtungen auf Facebook aktiv: $8 \%$ posteten gelegentlich Inhalte auf Facebook, $11 \%$ mehrmals im Monat und $3 \%$ mehrmals pro Woche (vgl. Abbildung 22).

Abbildung 22: Digitale Angebote: Postings auf Facebook

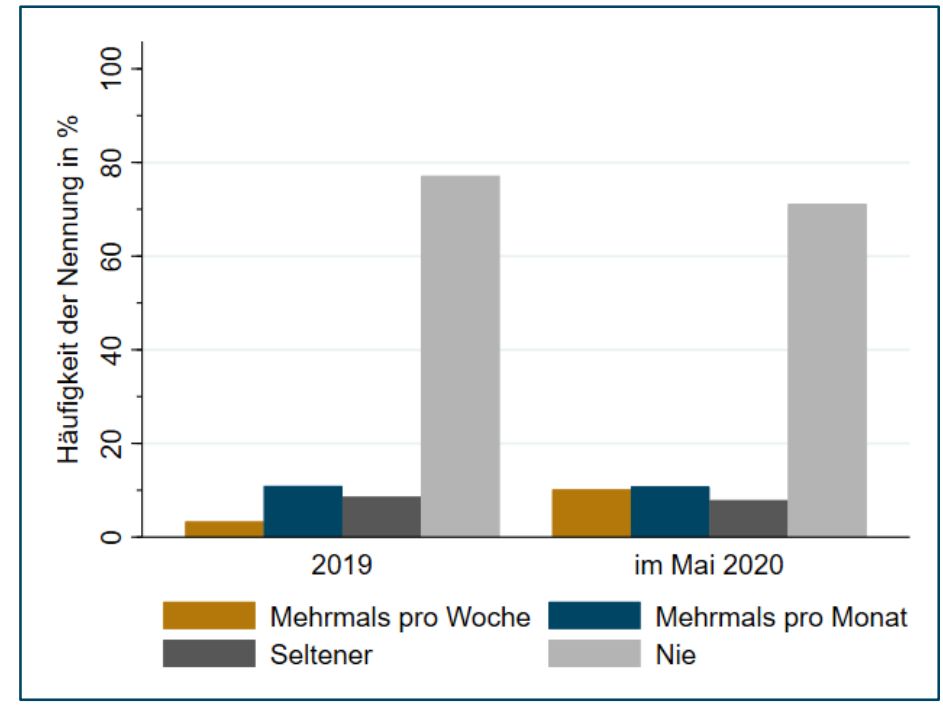

Quelle: ifb-Befragung „kontakt.los! 2020“ ( $n=402$; keine Angabe: "2019“ = 100; "im Mai 2020“ =97) 
Im Zuge der Corona-Pandemie wurde dieser Kanal ausgiebiger genutzt: $9 \%$ stellten gelegentlich Postings ein, $11 \%$ mehrmals pro Monat und $10 \%$ mehrmals pro Woche. Insgesamt waren $29 \%$ der Einrichtungen auf Facebook präsent und posteten auf diesem Wege Informationen zu Familien- und Corona-Themen sowie zu Aktivitäten der Einrichtung, stellten Spiel- und Bastel- sowie auch Erziehungstipps (häufiger auch im Video-Format) ein oder teilten Sprüche und Bilder (vgl. Abbildung 22).

Nur bei wenigen Einrichtungen zählen Postings auf der Plattform Instagram sowie die Produktion von Audio-Podcasts zum digitalen Repertoire. Deren Verbreitung nahm im Zuge der Pandemie aber jeweils leicht zu: Instagram wurde 2019 von rund $5 \%$ der Einrichtungen genutzt, im Mai 2020 von $9 \%$. Audio-Podcasts wurden vor der Pandemie von $2 \%$ der Einrichtungen erstellt, im Mai 2020 stieg dieser Anteil auf $6 \%$.

Etwas stärker verbreitet, zumindest seit Ausbruch der Corona-Pandemie, ist das Posten von Filmen und Video-Podcasts. Zwar wurde die Plattform YouTube im Jahr 2019 nur sehr vereinzelt von rund $1 \%$ der Einrichtungen und im Zuge der Pandemie auch nur von $4 \%$ genutzt. Bezieht man aber alle Filme und Video-Podcasts mit ein, also auch diejenigen, die beispielsweise auf der eigenen Webseite oder dem Facebook-Profil veröffentlicht wurden, so wird deutlich, dass Filme beliebter werden: Während im Jahr 2019 insgesamt knapp $4 \%$ der Einrichtungen dieses Medium nutzten, stieg dieser Wert im Zuge der Pandemie im Mai 2020 deutlich an: 7 \% posteten gelegentlich Filme oder Video-Podcasts, $6 \%$ mehrmals pro Monat und $4 \%$ gar mehrmals pro Woche.

\section{Einschränkungen und Barrieren}

Aufgrund der Corona-Pandemie konnten Angebote der Familienbildung und Beratung, die üblicherweise im persönlichen Kontakt stattfanden, nicht wie gewöhnlich durchgeführt werden. Sie mussten modifiziert und zum großen Teil auch komplett eingestellt werden. Bei welchen dieser Angebote, die nicht durchgeführt werden konnten, schätzen Fachkräfte dies als besonders bedauerlich ein? Und was waren Einschränkungen und Hürden, die eine Digitalisierung oder andere Modifizierung dieses Angebots verhindert haben?

Die Befragten wurden gebeten, ein konkretes Beispiel für ein etabliertes Angebot anzugeben, das sie derzeit nicht - auch nicht in modifizierter Form - anbieten können und dessen Aussetzung sie aus fachlicher Sicht als besonders bedauerlich einschätzen. Am häufigsten wurde bedauert, dass auf offene Treffs und Elterncafés verzichtet werden musste: Mehr als ein Viertel (28\%) der Befragten, insbesondere aus dem Bereich der Familienbildung, nannte dieses Format, welches sich als niedrigschwellige Kontakt- und Gesprächsmöglichkeit etabliert hat. Andere Fachkräfte bedauern insbesondere den Verzicht auf bestimmte Kurse (19\%), die nicht umsetzbaren Vorträge und Informationsveranstaltungen (13\%) sowie Eltern-(Kind-)Gruppen- oder Selbsthilfegruppen (12\%). Weitere Fachkräfte führten hier insbesondere den Ausfall aufsuchender Gruppenangebote an (12\%). Besonders häufig wurde dies von Beratungsstellen für Schwangerschaftsfragen genannt, deren Angebote der Sexualpädagogik an Schulen und anderen Einrichtungen abgesagt werden mussten. Andere Angebote, deren Wegfall stark bedauert wird, sind beispielsweise Beratungsgespräche, Hausbesuche, Workshops oder Feste (vgl. Abbildung 23). 
Abbildung 23: Als besonders bedauerlich bewertet wird die Absage von ...

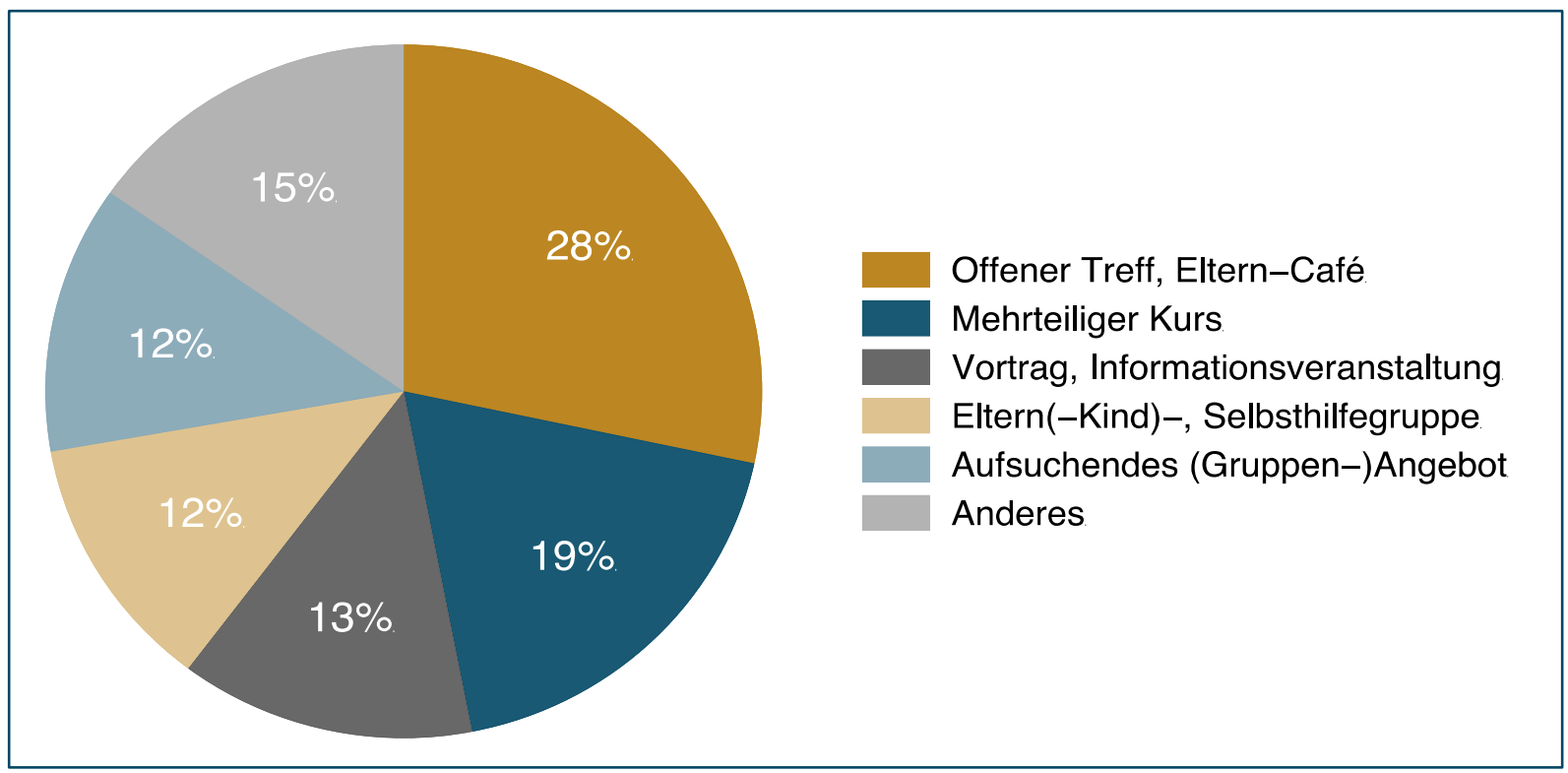

Quelle: ifb- Befragung „kontakt.los! 2020” ( $n=402$; keine Angabe = 97)

Als Grund, warum das jeweilige Angebot auch in veränderter Form nicht angeboten wird, wurde am häufigsten genannt, dass die derzeit gültigen Abstands- und Hygienevorschriften nicht eingehalten werden können (73\%), Alternativen wie die Durchführung des Angebots im Freien nicht möglich sind (29\%) und das Angebot grundsätzlich nicht digitalisierbar ist ( $23 \%$ ). Jeweils $6 \%$ der Fachkräfte gaben an, dass sie mehr Kompetenzen für die Digitalisierung dieses Angebotes benötigt hätten oder Datenschutzaspekte entgegenstünden (vgl. Abbildung 24).

Abbildung 24: Gründe für den Ausfall eines konkreten Angebotes: Bereich Digitalisierung

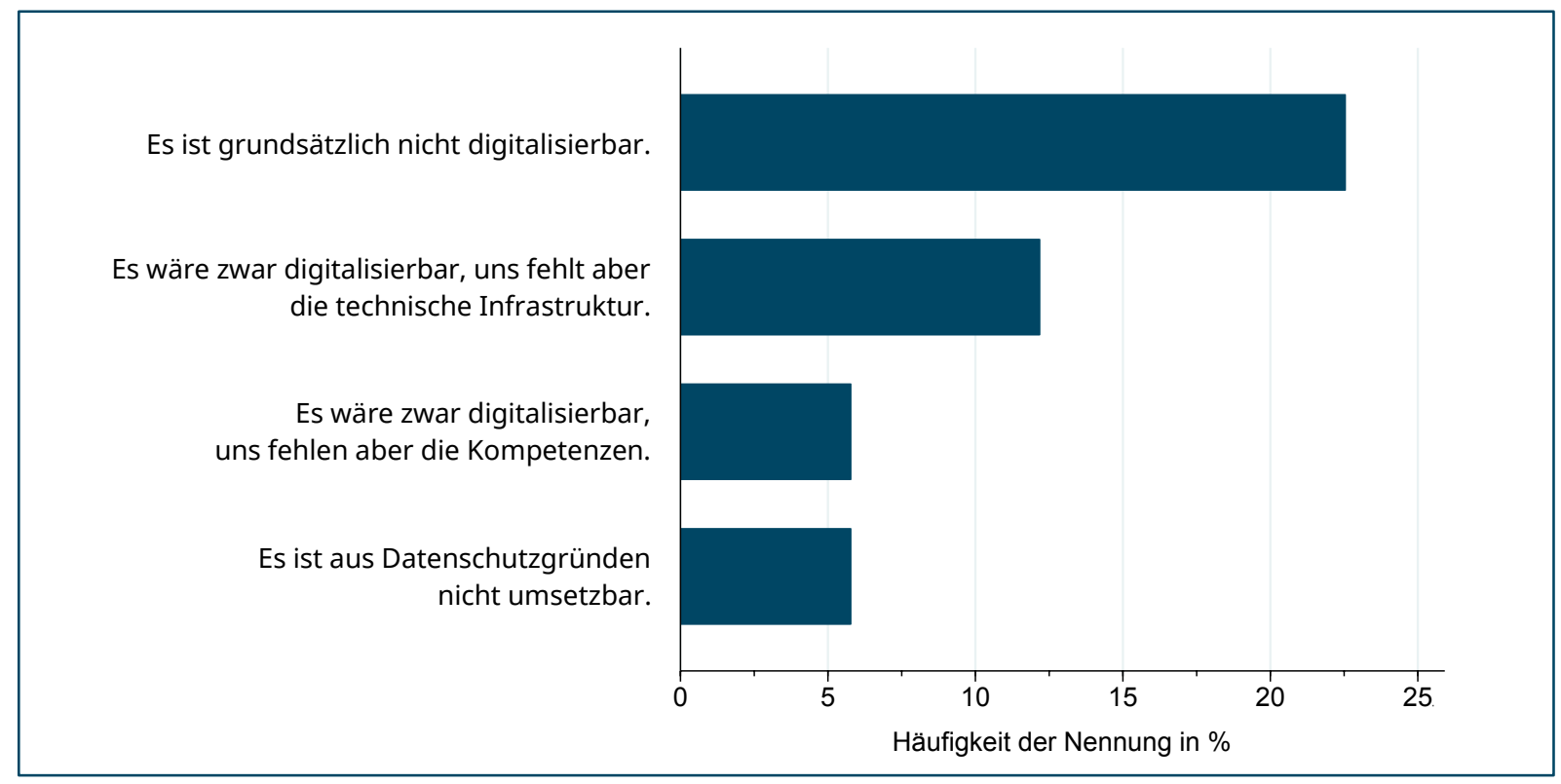

Quelle: ifb-Befragung "kontakt.los! 2020" ( $n=402$; keine Angabe = 74)

Anmerkung: Mehrfachnennungen möglich 
Von denjenigen, die ein beliebtes Angebot wegen fehlender technischer Infrastruktur nicht anpassen konnten (12\%), nannten fast alle, nämlich 38 von 40 Befragten, fehlende Hardware, 35 Personen nannten fehlende Software und knapp die Hälfte nannte das Nichtvorhandensein einer ausreichend schnellen und stabilen Internetverbindung als Gründe .

Ein oft genannter Grund für die Aussetzung des Angebots war außerdem, dass die Niedrigschwelligkeit bei einer Adaptierung an derzeitige Rahmenbedingungen verloren ginge (19\%). Auch in den offenen Antworten spiegeln sich Bedenken über zusätzliche Barrieren durch digitale Angebote wider, weil etwa "die Teilnehmenden die technischen Vorrausetzungen nicht haben (finanziell, sprachlich)" und "Menschen mit Lernbehinderung dazu nur schwer Zugang haben".

Wurde angegeben, dass ein Angebot grundsätzlich nicht digitalisierbar sei, wurde dies am häufigsten mit methodisch-didaktischen Gesichtspunkten begründet. Bei vielen Angeboten der Familienbildung ginge es beispielsweise um die direkten Interaktionen und den körperlichen Kontakt zwischen Eltern und Kind sowie Kindern untereinander. So bewerten Fachkräfte das Angebot als nicht digitalisierbar, weil "Rollenspiele live stattfinden müssen“. Ebenso wird zu bedenken gegeben, dass digitale Angebote für bestimmte Altersgruppen ungeeignet sind: "Säuglinge können nicht von PC-Angeboten partizipieren, es ist entwicklungspsychologisch nicht angeraten."

Bei einzelnen Angeboten ist evident, dass eine Anpassung nicht möglich war, da die entsprechende Umgebung nicht nutzbar war (beispielsweise beim Babyschwimmen), Bewegungen und Berührungen konzeptuell im Angebot verankert sind (z. B. faires Raufen), bestimmte Materialien gemeinsam genutzt werden oder bei Ernährungsthemen die gemeinsame Zubereitung von Essen im Mittelpunkt steht, was aus Infektionsschutzgründen zu dem Zeitpunkt verboten war.

Für viele Fachkräfte ist zudem die Anwesenheit der (werdenden) Eltern an einem gemeinsamen Ort besonders wichtig und eine notwendige Voraussetzung für das Gelingen konkreter Angebote. So schätzen zahlreiche Fachkräfte Angebote als grundsätzlich nicht digitalisierbar ein, weil "es von sozialer Interaktion lebt", "der Austausch unter den Teilnehmenden im Mittelpunkt steht", "es auch darum geht, sozialer Isolation vorzubeugen" oder weil der "persönliche Kontakt" und „echte Kontakte und Austausch der Eltern untereinander nur über Treffen möglich ist". Dies verdeutlicht einerseits den Stellenwert und die Qualität von Präsenzangeboten in der Arbeit mit Familien, gerade auch in der Arbeit mit Zielgruppen, für welche Beratungs- und Bildungsangebote grundsätzlich mit großen Hemmschwellen verbunden sind. Andererseits zeigen die Antworten aber auch, dass in unserer Gesellschaft virtuelle Treffen noch nicht breit akzeptiert, selbstverständlich und etabliert sind und erst seit Ausbruch der Corona-Pandemie rasant verstärkt beruflich wie privat zur Kommunikation, für Besprechungen und zum fachlichen oder privaten Austausch genutzt werden. 


\section{Good Practice: Erfahrungen während der Pandemie}

Gleichzeitig gab es zahlreiche Angebote, mit denen Einrichtungen gute Erfahrungen während der Corona-Pandemie machten. Diese hatten sie entweder bereits vor der Pandemie etabliert, an die neue Situation adaptiert oder ganz neu konzipiert: Hier nannten die Befragten sowohl Präsenzangebote vor Ort (10 \%) als auch Angebote im Freien (12\%) - und fast 250 Befragte (74 \%) nannten digitale Good Practices (vgl. Abbildung 25).

Abbildung 25: Good Practices während der Corona-Pandemie

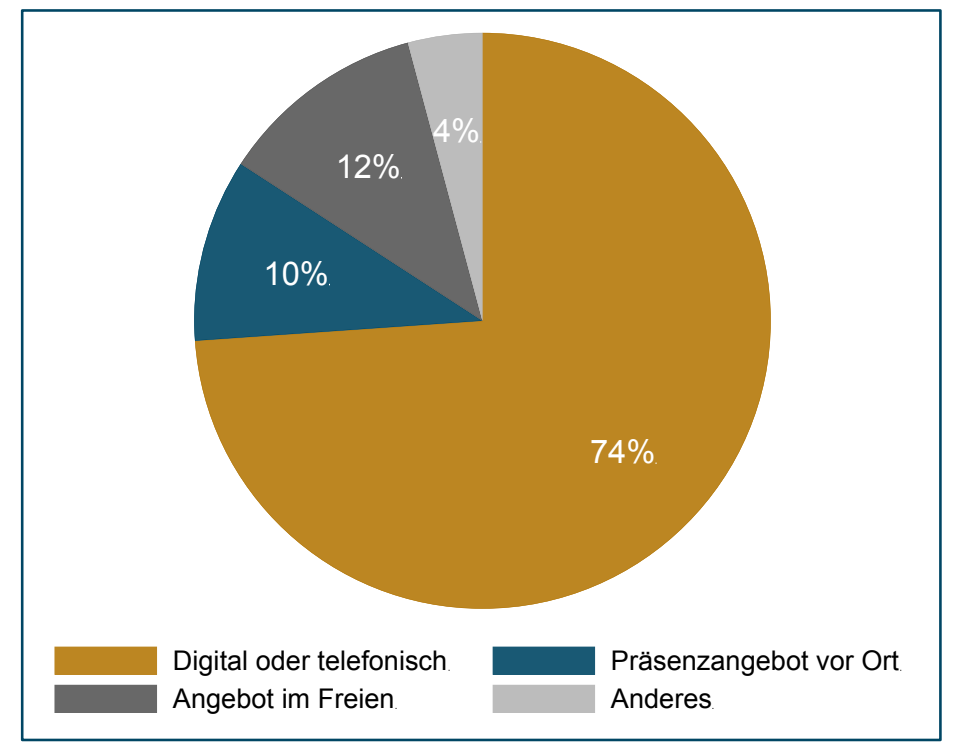

Quelle: ifb-Befragung „kontakt.los! 2020” ( $=402$; keine Angabe =76)

Zur Kategorie digitale Angebote zählen zum einen zahlreiche telefonische Angebote mit unterschiedlichem Fokus, auch außerhalb üblicher Sprechzeiten: z. B. Elterntelefon, Telefonsprechstunde, Sorgentelefon, Schlaf-Schrei-Sprechstunde, ErziehungsberatungsTelefon, Familienkrisentelefon-Hotlines oder "Gehstruktur per Telefon - Wir rufen unsere Besucherinnen $a n^{\prime \prime}$.

Zum anderen wurden auch modernere digitale Technologien für Angebote eingesetzt: Zahlreiche Beratungsstellen nutzten Video-Konferenzsoftware und ähnliche Tools, um Einzel- und Paarberatungen per Video durchzuführen, manche kombinierten beraterische Face-to-Face-Gespräche vor Ort mit Gesprächen via digitale Medien (z. B. „Embedded Counselling“ und „Blended Counselling“). Auch klassische und etablierte Präsenzangebote der institutionellen Familienbildung werden online durchgeführt. So gibt es beispielsweise Online-Mütter- oder Eltern-Gruppen, ein Babycafé, PEKiP online, Online-Musikgarten und -Singkreis oder auch einen Babymassage-Kurs via Skype. Werdende Eltern wurden per Webseminar auf die Geburt und das Leben als Familie vorbereitet, über familienunterstützende Leistungen informiert oder erhielten „Haushalts-Coaching“.

Mehrere nutzten auch Messenger-Dienste für Eltern-Chats, E-Mails für Newsletter (u. a. mit Spielangeboten und pädagogischen Empfehlungen) oder stellten Videos rund um das Thema "Coronavirus" in sozialen Netzwerken bereit. Eine Institution hat eine digitale Kontaktbörse gestartet, um Selbsthilfe zu aktivieren, Austausch zu fördern und sozialer Isolation vorzubeugen. 
Die während der Corona-Pandemie angebotenen digitalen (inkl. telefonischen) Angebote stellen für einen kleinen Teil eine Zwischenlösung dar: $12 \%$ verneinen die Frage, ob sie dieses Angebot auch nach der Corona-Pandemie gerne anbieten würden. $18 \%$ der Befragten können dies (noch) nicht abschätzen. Für sieben von zehn Einrichtungen (70 \%) hat sich ihr neues Angebot aber so bewährt, dass sie dieses auch nach der CoronaPandemie gerne weiterhin anbieten würden (vgl. Abbildung 26). ${ }^{11}$

Abbildung 26: Fortführung digitaler Good Practices auch nach der Corona-Pandemie

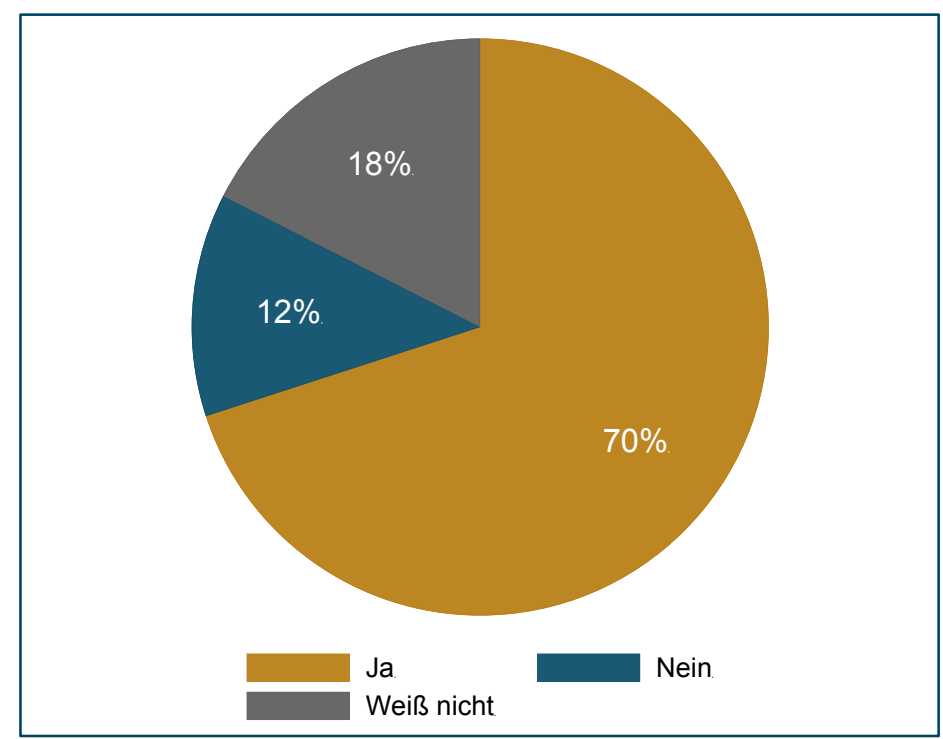

Quelle: ifb-Befragung „kontakt.los! 2020" (n= 402; "trifft nicht zu“ = 171)

Für die meisten Einrichtungen bedeutet die Corona-Pandemie somit, dass sie bewährte Angebote aussetzen müssen, weil diese unter den gegebenen Rahmenbedingungen nicht möglich und aus verschiedenen Gründen auch nicht digitalisierbar sind. Zugleich sind aber auch - oft unter Einsatz von großen Ressourcen und vor dem Hintergrund finanzieller Unsicherheit - zahlreiche gute Ideen im Bereich digitaler Angebote ausgebaut oder neu erarbeitet worden. Von Telefon- und Videoberatung über Rundmailings und Chats bis zu Webseminaren - die Mehrheit macht mit ganz verschiedenen Angeboten so gute Erfahrungen, dass sie diese auch in Zukunft fortsetzen möchten. Durch die CoronaPandemie und die Maßnahmen zum Infektionsschutz gab es im Bereich der Eltern- und Familienbildung, der Beratung und Frühen Hilfen einen eindeutigen Digitalisierungsschub.

11 Frage lautete: „Spontan: Würden Sie dieses Angebot gerne auch nach der Corona-Pandemie anbieten?“ 


\section{In Kontakt bleiben: 10 Aspekte}

Auf welche Weise ist es den Fachkräften trotz der Corona-Maßnahmen und all den damit verbundenen Herausforderungen besonders gut gelungen, mit ihrer Zielgruppe in Kontakt zu kommen oder zu bleiben?

Offenheit und Erreichbarkeit. So simpel es auch klingt: Es erwies sich erstens als wichtig und essenziell für die Einrichtungen in der Stichprobe, überhaupt weiterhin geöffnet zu haben und erreichbar zu sein. So betonen einige, dass sie ununterbrochen für die Familien da sein konnten („Die Beratungsstelle war zu keiner Zeit geschlossen. Die Frauen im Schwangerschaftskonflikt wurden alle beraten.") - und dies nach verantwortungsvollem Ermessen eben auch als Präsenzangebot. So schreibt eine Einrichtung, dass die telefonische Beratung von Klientinnen sehr gut angenommen wurde, aber dass „in Krisen-, Not-, und Konfliktfällen" auch die persönliche Beratung "immer möglich [war] nach vorheriger telefonischer Vereinbarung. Wir waren quasi eh nie weg. "Aufgrund der Hygieneschutz-Vorgaben sowie Ressourcenknappheit war es aber nicht allen Einrichtungen möglich, ihre Türen für Familien offen zu halten, sodass Schwangeren und Eltern nur ein eingeschränktes Angebot zur Verfügung stand.

Außenkommunikation und Präsenz. Zweitens war und ist wichtig, die Erreichbarkeit offensiv zu zeigen und breit gestreut darüber zu informieren, dass man weiterhin erreichbar ist sowie wann und wie man erreichbar ist. Diese Außenkommunikation gelingt zum einen über die verschiedenen (analogen und digitalen) Informationskanäle: Es wurde sowohl klassische Medienarbeit betrieben (z. B. Informationen zu Sprechzeiten in der lokalen Tageszeitung publiziert) als auch die eigene Homepage genutzt und soziale Medien bespielt, insbesondere Facebook und Messenger-Broadcast-Listen. Bei dieser Öffentlichkeitsarbeit auch die Niedrigschwelligkeit darzustellen und auf die Schweigepflicht der Einrichtungen hinzuweisen, sei sinnvoll, wie eine Fachkraft erklärt. Zum anderen sind die physische Präsenz und das Schaffen von Gelegenheitsstrukturen bedeutsame Erfolgsfaktoren, um die Zugänglichkeit weiterhin zu gewährleisten: Eine Fachkraft berichtet, dass sie es besonders gut geschafft hat, Kontakt zu halten, indem sie jeden Tag vor Ort und präsent war - und dies auch bewusst gezeigt und forciert hat: „Mein Büro habe ich teilweise in unseren Garten verlegt, damit die Leute mich sehen können. Da viele Stammbesucher*innen an unserem Haus vorbeikommen, bin ich viel mit ihnen in Kontakt gekommen."

Unkonventionell und unkompliziert: analoge Ideen. Drittens wurde auf kreative Weise der Kontakt zu Familien bewusst initiiert: So wurden Bastel- oder Spielmaterialien zum Mitnehmen an den Einrichtungen ausgegeben, um Familien Anregungen für daheim mitzugeben - und damit sich beim Abholen unkomplizierte „Fenstergespräche“ ergeben können. Andersherum wurden Anlässe für aufsuchende Arbeit gestaltet, um sich gezielt nach dem Wohl von Eltern und Kindern zu erkundigen. Beispielsweise wurden Alltagsmasken genäht und bei Familien vorbeigebracht, Osterbesuche abgestattet sowie persönliche Postkarten, Brief- und Paketbotschaften (z. B. in Form einer "Vergesst-unsnicht-Tüte") bei Familien abgegeben, damit der Kontakt aufrecht erhalten blieb sowie Eltern darauf aufmerksam gemacht und daran erinnert wurden, dass sie sich weiterhin an die Fachkräfte wenden können und diese bei Bedarf unterstützend tätig werden. Des Weiteren wurden vereinzelte Gruppenangebote ins Freie verlegt. Auch Beratungs- 
Spaziergänge („Walk \& Talk“) erwiesen sich für viele Institutionen als gelingendes Angebot - nach Terminvereinbarung oder auch spontan, und sogar außerhalb der eigentlichen Arbeitszeiten und -orte („Außerdem habe ich auch auf dem Weg in die Arbeit, auf dem Heimweg oder bei der Mittagspause im nahegelegenen Park immer viele Familien angetroffen, mit denen ich viele Gespräche geführt habe."). Solche unkonventionellen und niedrigschwelligen Ansätze funktionieren dann besonders gut, wenn das Vertrauen der Familien in Mitarbeiterinnen und Mitarbeiter und die Einrichtung bereits im Vorfeld erarbeitet werden konnte, wie eine Fachkraft anmerkt. Und sie gelingen dann, wenn im Team ausreichend Ressourcen zur Verfügung stehen, die Arbeitsbedingungen flexibel und die Atmosphäre inspirierend sind: "Die Flexibilität des Teams und die hohe Bereitschaft erreichbar zu sein, hat personelle Engpässe (Kinderbetreuung usw.) kompensiert und viele Ideen frei gesetzt (Beratungs-Spaziergänge, gemeinsam online Anträge ausfüllen, umfänglicher Schriftverkehr, wenn Menschen digital nicht erreichbar sind und selbst nicht in die Beratungsstelle kommen möchten)." Teilweise wurden dabei auch Maßnahmen umgesetzt, die über das engere Verständnis des Bildungs- und Beratungsauftrags hinausgehen, für benachteiligte Familien aber immens wichtig und für die Einrichtungen Türöffner waren: Unterstützung von Home-Schooling durch das Ausdrucken von Schulmaterialien (für Familien ohne entsprechende Technik) und PC-Support sowie Unterstützung bei der Kommunikation mit der Schule.

Netzwerk und Zusammenarbeit. Das eigene professionelle Netzwerk und der Weg über Multiplikatorinnen und Multiplikatoren haben während der Krise für einige Befragte nicht so gut funktioniert wie üblicherweise, weil mehrere Stellen nicht zugänglich, nicht verfügbar oder schlechter erreichbar waren. Für andere erwiesen sich diese Netzwerke hingegen als hilfreiche Ressource, auf die in der Zeit um den Lockdown gebaut werden konnte, um Ratsuchende in weiterführende Angebote vermitteln zu können oder um die Zugänge zur eigenen Einrichtung zu erleichtern. Daher gilt, viertens, als eine von vielen Maßnahmen, die dazu führen, dass Familien sich tatsächlich Hilfe in Einrichtungen holen: "Netzwerke intensivieren: Schulsozialarbeit, Bibliothek ..., unter dem Blickwinkel: Wie können wir gemeinsam die Familien erreichen, die Hilfe brauchen?"

Das gute alte Telefon. Besonders häufig wird, fünftens, berichtet, dass telefonische Angebote wie Telefon-Hotlines und telefonische Beratungen massiv ausgebaut wurden. Die „Familien freuten sich über Anrufe - und hatten dann sehr häufig das Bedürfnis, zu erzählen/berichten, wie es ihnen geht. Besonders wichtig in dieser Zeit - einfach Zuhören“. Dies gilt auch und gerade für belastete Familien. Als erfolgreich bewertet wurde die "aktive Kontaktaufnahme mit potentiell besonders betroffenen bereits bestehenden Klienten per Mail oder Telefon. Das ist normalerweise nicht üblich." Problemen kann so aber vorgebeugt, und bestehende Probleme können schneller erkannt werden.

Digitale Angebote. Sechstens betonen zahlreiche Einrichtungen den Wert der digitalen Formate. Es gab eine breite Palette, wie oben bereits erläutert wurde: One-way-Kommunikation sowie interaktive Angebote, solche via E-Mail, Video-Telefonie und -Chat, informierende, beratende sowie anregende, und kostenpflichtige sowie kostenfreie Angebote. Gerade das „Bündel von [digitalen] Angeboten und die sehr schnelle Implementierung nach der Schließung“ habe bei einigen Einrichtungen zum Erfolg geführt: „Beratungstelefone für Erziehung und Schwangerschaft, Anregungen auf Facebook zur Alltagsgestaltung, Erziehungstipps auf Facebook, Fortführung von Kursen über kostenlose Chats. [In einem] 2. 
Schritt: Ergänzung E-Mail-Beratung (...), kostenpflichtige länger laufende Kurse und Infoveranstaltungen über (...) Video-Konferenz-Software und YouTube-Videos." Manche Einrichtungen konnten hier auf ihren Erfahrungsschatz zurückgreifen, andere haben sich neu eingearbeitet: „Wir haben neue Formate entdeckt und gut genutzt, die wir auch in der Zukunft anbieten werden. Wir werden weiter dran arbeiten, dass uns neue Formate, auch Webinare, Videotools etc., künftig zur Verfügung stehen. Durch die Krise gab es einen Digitalisierungsschub und die Erfahrung der Vorteile dadurch. D. h. die Angebote für die Zielgruppen werden sich langfristig erweitern." Gelobt wurden in diesem Kontext manche Träger, welche schnelle digitale Lösungen zur Durchführung von Videoberatungen zur Verfügung stellten, die beeindruckenden ehrenamtlich "tätigen Kind-Gruppenleiterinnen, Tageseltern und Referent*innen mit einer tollen Bereitschaft, sich zu engagieren und dazu zu lernen" wie auch die Aufgeschlossenheit der Klientinnen und Klienten, an dieser neuen Form der Kommunikation teilzunehmen. So vielversprechend solche Zitate auch klingen, so nüchtern muss zugleich festgehalten werden, dass diese eine Ausnahme darstellen - was sich mit den oben dargestellten Ergebnissen zur Verbreitung digitaler Angebote deckt. Viele Einrichtungen haben erste Ansätze ausprobiert; bisher mangelt es jedoch an technischer Infrastruktur (Hard- und Software, Lizenzen) und insbesondere Erfahrungswerten sowie an zeitlichen Ressourcen, um entsprechende Angebote umfassend neu konzipieren, bewerben und umsetzen zu können.

Methodenvielfalt. Siebtens stellt vor allem die Kombination verschiedener Angebotsformate, Zugangswege und Kommunikationskanäle für auffallend viele Fachkräfte einen Erfolgsfaktor dar. Hiermit ist zum einen gemeint, verschiedene Formate, Wege und Kanäle parallel zu nutzen: Besonders gut sei der Kontakt dadurch gelungen, berichten mehrere Befragte, dass den Betroffenen die Wahlmöglichkeit gegeben werden konnte, ob sie per Telefon, per Video oder durch Präsenzangebote in Kontakt treten wollen. So können Einrichtungen den unterschiedlichen Bedürfnissen der unterschiedlichen Eltern gerecht werden. („Der Methodenmix hat uns ermöglicht, uns auf unterschiedliche Bedürfnisse und Situationen einzustellen.") Zum anderen ist gemeint, unterschiedliche Methoden und Medien zu einem neuen Angebotsformat zusammenzubringen: „Für die Beratung erwies sich ein Blended Counselling-Modell als positiv", beispielsweise durch eine Kombination von Gesprächen vor Ort (üblicherweise das Erstgespräch oder Gespräche in akuten Krisensituationen) mit Beratungsgesprächen via Video-Telefonie oder anderen digitalen Medien. Diejenigen Einrichtungen, die schon vor der Krise entsprechend umgestellt hatten, waren auf ein ergänzendes digitales Arbeiten naturgemäß besser vorbereitet als andere.

Neue Themen erkennen und setzen. Nicht nur bezüglich der Zugangswege und Methoden war Flexibilität gefragt, sondern, achtens, auch aufgrund der teils neuen und vielschichtigen Anliegen. So wurden Online-Workshops zu aktuell relevanten Themen angeboten, beispielsweise „zum Thema Werte in der Erziehung, da sich viele Familien neu orientiert haben, sich neu aufstellen mussten“. Auch mussten sich Fachkräfte auf neue Themen der Beratungsgespräche einlassen und auf die oben geschilderten Mehrfachbelastungen und komplexe Problemlagen adäquat eingehen können. Als erfolgreich genannt wurde beispielsweise die „Unterstützung bei Entwicklung von Tagesplänen“, um all die Herausforderungen von Home-Schooling, Home-Office und Beschäftigung der Kleinkinder unter einen Hut zu bekommen. Dabei wurden die Familien auch angeregt, an "Schönes und Tages-Highlights, nach draußen gehen ..." zu denken. Auch die Vermittlung von 
Faktenwissen über das Corona-Virus (z. B. Verbreitung und Gefahren) wurde als wichtig erachtet, nicht nur für eigene Hygienekonzepte, sondern auch für deren Vermittlung an besorgte Eltern, insbesondere „für Familien, die das Haus wochenlang gar NICHT mehr verlassen haben".

Nicht immer zufriedenstellend. Allen positiven Beiträgen zum Trotz: Die Voraussetzungen der einzelnen Einrichtungen sind extrem unterschiedlich, wie oben beschrieben. Und zahlreiche Einrichtungen berichten, dass sie kaum oder gar nicht in Kontakt mit Familien bleiben konnten („Es ist uns leider nicht gelungen.") Und selbst diejenigen, denen es gut gelungen ist, in Kontakt zu bleiben, mussten Abstriche machen; sie haben deutlich weniger Menschen erreicht als üblich. In besonderem Ausmaß gilt dies zum einen für Familien, mit denen bisher noch keine Beziehung bestand, auf der man hätte aufbauen können: „Besonders gut gelungen ist es nicht. Die Familien, die bereits gut bei uns angebunden waren, konnten per Telefon und auf dem Postweg relativ gut versorgt werden. Neue Klienten kamen deutlich weniger. Beratungen mit neuen Klienten waren deutlich kürzer. Telefonische Beratungen waren für Fachkräfte deutlich aufwendiger und weniger zufriedenstellend." Zum anderen ist es ausgerechnet bei Familien mit Belastungen schwierig, den Kontakt aufzubauen und zu halten. Der Kontakt zu Alleinerziehenden, zu Familien mit Migrationshintergrund (vor allem Geflüchteten) sowie zu bildungsinstitutionenfernen und zu belasteten Familien ist erschwert - und in vielen Fällen abgerissen, denn für stark belastete Familien sind Angebote in der Corona-Zeit mit noch mehr Barrieren verbunden als sonst. Gefährdete Familien und Kinder sind häufig gar "von der Bildfläche verschwunden", sodass es für die Expertinnen und Experten schwieriger bis unmöglich ist, Risiken im Blick zu behalten und potenzielle Kindswohlgefährdungen zu erkennen. Aufgrund der Corona-Pandemie, so eine öfter getätigte Aussage, „werden Benachteiligte weiter abgehängt".

Kontakt braucht Ressourcen. Viele Institutionen, vor allem die präventiv tätigen Familienbildungseinrichtungen, hatten finanzielle Einbußen und mussten (Honorar-) Verträge aussetzen; manche der befragten gemeinnützigen Organisationen plagen gar Existenzängste. Zugleich wurde der coronabedingte höhere Arbeitsaufwand von mehreren Stimmen thematisiert - wie auch die fehlende Wirtschaftlichkeit: „Enormer Arbeitsaufwand - digitaler und telefonischer Kontakt zu Teilnehmer*innen, Kolleg*innen, Referent*innen - viel mehr Zeit für Gespräche am Telefon. Ich war teilweise am Limit meiner eigenen Ressourcen! Aber, es hat sich gelohnt - sicher nicht finanziell ..." Für einen guten Kontakt zu den Menschen, für einen wertschätzenden Dialog und eine fundierte Beratung brauche es eben vor allem Zeit, wie einige Fachkräfte hervorheben. Und: „Familienbildung lebt vom lebendigen Austausch in Gruppen vor Ort. Online-Kurse sind kein Ersatz." Aber wie die Erfahrungen der Fachpraxis in der Corona-Pandemie zeigen, können digitale Formate genau wie auch unkonventionelle analoge Formate das bereits etablierte Angebotsspektrum von Familienbildungseinrichtungen, Beratungsstellen sowie der KoKis gewinnbringend ergänzen. Mit ausreichenden Ressourcen kann dies nachhaltig umgesetzt werden. 


\section{Literaturverzeichnis}

Andresen, Sabine/Lips, Anna/Möller, Renate/Rusack, Tanja/Schröer, Wolfgang/Thomas, Severine/Wilmes, Johanna (2020): Kinder, Eltern und ihre Erfahrungen während der Corona-Pandemie. Hildesheim. doi:10.18442/121

Bayerisches Staatsministerium für Familie, Arbeit und Soziales [StMAS] (2020): Neues Onlineportal zu Hilfsangeboten bei häuslicher Gewalt. Pressemitteilung vom 10.04.2020. Online verfügbar unter https://www.stmas.bayern.de/aktuelle-meldungen/pm2004-095.php [aufgerufen am 03.08.2020]

Bünning, Mareike/Hipp, Lena/Munnes, Stefan (2020): Erwerbsarbeit in Zeiten von Corona. WZB Ergebnisbericht. Online verfügbar unter https://www.econstor.eu/bitstream/10419/216101/1/Full-text-report-Buenning-et-al-Erwerbsarbeit-inZeiten-von-Corona-v1-20200415.pdf [aufgerufen am 03.08.2020]

Deutscher Ethikrat (2020): Solidarität und Verantwortung in der Corona-Krise. Ad-hocEmpfehlung. Online verfügbar unter https://www.medrecherche.de/Deutscher Ethikrat 27.03.2020.pdf [aufgerufen am 03.08.2020]

Erziehungshilfefachverbände (2020): Dringender Handlungsbedarf bei Sicherstellung des Kinderschutzes in Zeiten von Corona (Covid-19). Zwischenruf der Erziehungshilfefachverbände. Online verfügbar unter https://afet-ev.de/veroeffentlichungen/Stellungnahmen/PDF-STellungnahmen/2020/200402--Zwischenruf-der-Erziehungshilfefachverbaende-zum-Kinderschutz-in-Zeiten-von-Corona..pdf?m=1585822201\& [aufgerufen am 03.08.2020]

Fatke, Bastian/Hölzle, Patricia/Frank, Andreas/Förstl, Hans (2020): Psychische Probleme in der Pandemie. Beobachtungen während der COVID-19-Krise. Deutsche Medizinische Wochenschrift 145, 10, S. 675-681. doi:10.1055/a-1147-2889

Mairhofer, Andreas/Peucker, Christian/Pluto, Liane/van Santen, Eric/Seckinger, Mike (2020): Kinder- und Jugendhilfe in Zeiten der Corona-Pandemie. DJI-Jugendhilfeb@rometer bei Jugendämtern. Deutsches Jugendinstitut: München. Online verfügbar unter https://www.dji.de/fileadmin/user upload/bibs2020/1234 DJI-Jugendhilfebarometer Corona.pdf [aufgerufen am 03.08.2020]

Steinert, Janina/Ebert, Cara (2020): Gewalt an Frauen und Kindern in Deutschland während COVID-19-bedingten Ausgangsbeschränkungen: Zusammenfassung der Ergebnisse. München. Online verfügbar unter https://drive.google.com/file/d/19Wqpby9nwMNjdgO4 FCqqlfyLLmBn7y/view [aufgerufen am 03.08.2020]

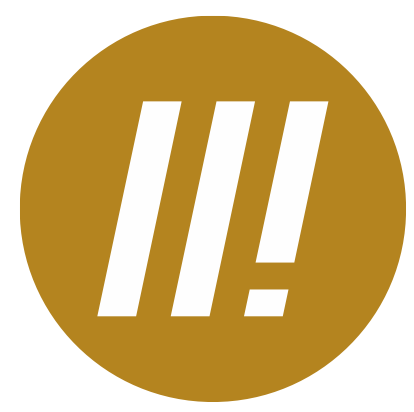

